\title{
WEIGHTED ALPERT WAVELETS
}

\author{
ROB RAHM, ERIC T. SAWYER ${ }^{\dagger}$, AND BRETT D. WICK ${ }^{\ddagger}$
}

\begin{abstract}
In this paper we construct a wavelet basis in $L^{2}\left(\mathbb{R}^{n} ; \mu\right)$ possessing vanishing moments of a fixed order for a general locally finite positive Borel measure $\mu$. The approach is based on a clever construction of Alpert in the case of Lebesgue measure that is appropriately modified to handle the general measures considered here. We then use this new wavelet basis to study a two-weight inequality for a general CalderónZygmund operator on $\mathbb{R}$ and conjecture that under suitable natural conditions, including a weaker energy condition, the operator is bounded from $L^{2}(\mathbb{R} ; \sigma)$ to $L^{2}(\mathbb{R} ; \omega)$ if certain stronger testing conditions hold on polynomials. An example is provided showing that this conjecture is logically different than existing results in the literature.
\end{abstract}

\section{Contents}

1. Introduction and statement of main results

2. Weighted Haar bases

2.1. Derivation of the explicit formula (2.2)

2.2. The Haar degenerate case

2.3. The global case

2.4. The higher dimensional case

3. Weighted Alpert wavelets with higher vanishing moments

3.1. Proof of Theorem 1

3.2. Uniqueness and degeneracy

3.3. Proof of Theorem 2

3.4. The special case $n=1$ and $k=2$

4. Application: a two weight $T p$ Conjecture

4.1. The Monotonicity Lemma

4.2. Comparison of $k$-energy and the usual 1-energy

4.3. An example with $\mathcal{A}_{2}^{0}<\infty, \mathcal{E}_{2, k}^{0}<\infty$ and $\mathcal{E}_{2,1}^{0}=\infty$

4.4. A Calderón-Zygmund operator satisfying testing conditions

References

\section{INTRODUCTION AND STATEMENT OF MAIN RESULTS}

The use of weighted Haar wavelet expansions has its roots in connection with the $T b$ theorem in DaJoSe] and CoJoSe, and came to fruition in treating the two weight norm inequality for the Hilbert transform in [NTV4, [Vol, the two part paper [LaSaShUr3], [Lac and [Hyt2]. The key features of the weighted Haar expansion $\left\{h_{I}^{\mu}\right\}_{I \in \mathcal{D}}$ are threefold:

(1) The Haar functions $\left\{h_{I}^{\mu}\right\}_{I \in \mathcal{D}}$ form an orthonormal basis of $L^{2}(\mu)$ :

$$
\begin{aligned}
& f=\sum_{I \in \mathcal{D}}\left\langle f, h_{I}^{\mu}\right\rangle_{L^{2}(\mu)} h_{I}^{\mu} \text { both pointwise } \mu \text {-a.e. and in } L^{2}(\mu), \\
& \text { where }\left\langle h_{J}^{\mu}, h_{I}^{\mu}\right\rangle_{L^{2}(\mu)}=\delta_{I}^{J},
\end{aligned}
$$

Date: May 20, 2019.

$\dagger$ Research supported in part by a grant from the National Science and Engineering Research Council of Canada.

$\ddagger$ Research supported in part by National Science Foundation DMS grants \# 1560955 and 1800057 . 
(2) Telescoping identities hold:

$$
\mathbf{1}_{K} \sum_{I \in \mathcal{D}: K \varsubsetneqq I \subset L}\left\langle f, h_{I}^{\mu}\right\rangle_{L^{2}(\mu)} h_{I}^{\mu}=E_{K}^{\mu} f-E_{L}^{\mu} f, \quad K \varsubsetneqq L,
$$

(3) Moment vanishing conditions hold:

$$
\int h_{I}^{\mu}(x) d \mu(x)=0, \quad I \in \mathcal{D} \text {. }
$$

In the setting of Lebesgue measure, Alpert Alp introduced new wavelets with more vanishing moments in (3), while retaining orthonormality (1) and telescoping (2). The expense of imposing these extra moment conditions is that one requires additional functions in order to obtain the expansion. The purpose of this note is to extend existence of Alpert wavelets to arbitrary locally finite positive Borel measures in Euclidean space $\mathbb{R}^{n}$, and to investigate degeneracy and uniqueness in the one-dimensional case as well. To state the main result in this paper requires some notation.

Let $\mu$ be a locally finite positive Borel measure on $\mathbb{R}^{n}$, and fix $k \in \mathbb{N}$. For $Q \in \mathcal{P}^{n}$, the collection of cubes with sides parallel to the coordinate axes, denote by $L_{Q ; k}^{2}(\mu)$ the finite dimensional subspace of $L^{2}(\mu)$ that consists of linear combinations of the indicators of the children $\mathfrak{C}(Q)$ of $Q$ multiplied by polynomials of degree at most $k-1$, and such that the linear combinations have vanishing $\mu$-moments on the cube $Q$ up to order $k-1$ :

$$
L_{Q ; k}^{2}(\mu) \equiv\left\{f=\sum_{Q^{\prime} \in \mathfrak{C}(Q)} \mathbf{1}_{Q^{\prime}} p_{Q^{\prime} ; k}(x): \int_{Q} f(x) x_{i}^{\ell} d \mu(x)=0, \quad \text { for } 0 \leq \ell \leq k-1 \text { and } 1 \leq i \leq n\right\}
$$

where $p_{Q^{\prime} ; k}(x)=\sum_{\alpha \in \mathbb{Z}_{+}^{n}:|\alpha| \leq k-1} a_{Q^{\prime} ; \alpha} x^{\alpha}$ is a polynomial in $\mathbb{R}^{n}$ of degree $|\alpha|=\alpha_{1}+\ldots+\alpha_{n}$ at most $k-1$. Here $x^{\alpha}=x_{1}^{\alpha_{1}} x_{2}^{\alpha_{2}} \ldots x_{n}^{\alpha_{n}}$. Let $d_{Q ; k} \equiv \operatorname{dim} L_{Q ; k}^{2}(\mu)$ be the dimension of the finite dimensional linear space $L_{Q ; k}^{2}(\mu)$.

Now define

$$
\begin{gathered}
\mathcal{F}_{\infty}^{k}(\mu) \equiv\left\{\alpha \in \mathbb{Z}_{+}^{n}:|\alpha| \leq k-1: x^{\alpha} \in L^{2}(\mu)\right\} \\
\text { and } \mathcal{P}_{\mathbb{R}^{n}}^{k}(\mu) \equiv \operatorname{Span}\left\{x^{\alpha}\right\}_{\alpha \in \mathcal{F}_{\infty}^{k}} .
\end{gathered}
$$

Let $\triangle_{Q ; k}^{\mu}$ denote orthogonal projection onto the finite dimensional subspace $L_{Q ; k}^{2}(\mu)$, let $\mathbb{E}_{Q ; k}^{\mu}$ denote orthogonal projection onto the finite dimensional subspace

$$
\operatorname{Span}\left\{\mathbf{1}_{Q}(x) x^{\alpha}: 0 \leq|\alpha| \leq k-1\right\}
$$

and let $\triangle_{\mathbb{R}^{n} ; k}^{\mu}$ denote orthogonal projection onto $\mathcal{P}_{\mathbb{R}^{n}}^{k}(\mu)$. The projections $\triangle_{Q ; k}^{\mu}$ are often referred to as multiresolution projections.

The first of two main results proved in this note is the following theorem, which establishes the existence of Alpert wavelets in all dimensions having the three important properties of orthogonality, telescoping and moment vanishing.

Theorem 1 (Weighted Alpert Bases). Let $\mu$ be a locally finite positive Borel measure on $\mathbb{R}^{n}$, fix $k \in \mathbb{N}$, and fix a dyadic grid $\mathcal{D}$ in $\mathbb{R}^{n}$.

(1) Then $\left\{\triangle_{\mathbb{R}^{n} ; k}^{\mu}\right\} \cup\left\{\triangle_{Q ; k}^{\mu}\right\}_{Q \in \mathcal{D}}$ is a complete set of orthogonal projections in $L_{\mathbb{R}^{n}}^{2}(\mu)$ and

$$
\begin{aligned}
f= & \triangle_{\mathbb{R}^{n} ; k}^{\mu} f+\sum_{Q \in \mathcal{D}} \triangle_{Q ; k}^{\mu} f, \quad f \in L_{\mathbb{R}^{n}}^{2}(\mu), \\
& \left\langle\triangle_{\mathbb{R}^{n} ; k}^{\mu} f, \triangle_{Q ; k}^{\mu} f\right\rangle=\left\langle\triangle_{P ; k}^{\mu} f, \triangle_{Q ; k}^{\mu} f\right\rangle=0 \text { for } P \neq Q,
\end{aligned}
$$

where convergence in the first line holds both in $L_{\mathbb{R}^{n}}^{2}(\mu)$ norm and pointwise $\mu$-almost everywhere.

(2) Moreover we have the telescoping identities

$$
\mathbf{1}_{Q} \sum_{Q \varsubsetneqq I \subset P} \triangle_{I ; k}^{\mu}=\mathbb{E}_{Q ; k}^{\mu}-\mathbb{E}_{P ; k}^{\mu} \quad \text { for } P, Q \in \mathcal{D} \text { with } P \varsubsetneqq Q
$$


(3) and the moment conditions

$$
\int_{\mathbb{R}^{n}} \triangle_{Q ; k}^{\mu} f(x) x^{\alpha} d \mu(x)=0, \quad \text { for } Q \in \mathcal{D}, 0 \leq|\alpha| \leq k-1 .
$$

In the special case of dimension $n=1$, we further investigate uniqueness and degeneracy of the wavelets constructed in Theorem 11. The system of one-dimensional Alpert wavelets is underdetermined, in general having $\left(\begin{array}{l}k \\ 2\end{array}\right)$ additional degrees of freedom which can be used to impose additional moment conditions in (3). The degeneracy condition for Alpert wavelets is phrased in terms of a matrix of moments $\boldsymbol{M}_{Q, k}$ and positive semi-definiteness, and can be interpreted as the degree to which $\mu$ is a finite sum of point mass measures within a given child of a cube. Here is our second main result, which includes the main points of our investigation into uniqueness and degeneracy, but not all of them - see Subsection 3.4 below for more. Let

$$
\boldsymbol{M}_{Q, k}=\int_{Q}\left[\begin{array}{ccccc}
1 & x & \cdots & x^{k-2} & x^{k-1} \\
x & x^{2} & \ddots & x^{k-3} & x^{k-2} \\
\vdots & \ddots & \ddots & \ddots & \vdots \\
x^{k-2} & x^{k-3} & \ddots & x^{2 k-4} & x^{2 k-3} \\
x^{k-1} & x^{k-2} & \cdots & x^{2 k-3} & x^{2 k-2}
\end{array}\right] d \mu(x)
$$

be the symmetric matrix of moments of the measure $\mu$ up to order $k-1$ on the interval $Q$.

Theorem 2. Let $\mu$ be a locally finite positive Borel measure on $\mathbb{R}$, fix $k \in \mathbb{N}$, and fix a dyadic grid $\mathcal{D}$ in $\mathbb{R}$. Then, in addition to parts (1) and (2) of Theorem 1 (restricted to $n=1$ ), we also have:

(1) The dimension of $L_{Q ; k}^{2}(\mu)$ is given by

$$
\operatorname{dim} L_{Q ; k}^{2}(\mu)=\operatorname{dim}\left(\text { Range } \boldsymbol{L}_{Q, k} \bigcap \text { Range } \boldsymbol{R}_{Q, k}\right),
$$

where $\boldsymbol{L}_{Q, k}$ and $\boldsymbol{R}_{Q, k}$ denote the matrices $\boldsymbol{M}_{Q_{\text {left }, k}}$ and $\boldsymbol{M}_{Q_{\mathrm{right}}, k}$ respectively and $Q_{\text {left }} / Q_{\text {right }}$ are the left and right halves of the interval $Q$. This shows in particular that $L_{Q ; k}^{2}(\mu)=k$ for all dyadic intervals $Q$ if and only if $\boldsymbol{M}_{Q, k} \succ 0$ for all dyadic intervals $Q$.

(2) In the case when $M_{Q, k} \succ 0$ for all dyadic intervals $Q$, we can choose an orthonormal basis $\left\{a_{Q}^{\mu, \ell}\right\}_{\ell=1}^{k}$ of $L_{Q ; k}^{2}(\mu)$ so that in addition to the moment conditions given in part (3) of Theorem 1 , the following additional moment conditions hold:

$$
\int a_{Q}^{\mu, \ell}(x) x^{i} d \mu(x)=0, \quad \text { for all } 2 \leq \ell \leq k \text { and } k \leq i \leq k+\ell-2 .
$$

The additional moment conditions in part (2) consume the remaining $\left(\begin{array}{c}k \\ 2\end{array}\right)$ degrees of freedom available in defining the $2 k^{2}$ coefficients in the functions $\left\{a_{Q}^{\mu, \ell}\right\}_{\ell=1}^{k}$, which we refer to as Alpert functions.

Remark 3. There is an analogous theorem in higher dimensions $n>1$, whose formulation and proof we leave for the interested reader.

When $k=1$, these theorems reduce to the well-known weighted Haar basis in $L^{2}(\mu)$ which is recalled in detail in Section 2. However, when $k \geq 2$, new wavelet bases are provided by this construction, and in Section 3, we prove our two main results regarding these new bases, Theorems 1] and 2, In the final section of this paper, we use the Alpert basis to study weighted inequalities for Calderón-Zygmund operators on the real line. A natural proof strategy is to decompose $f \in L^{2}(\sigma)$ and $g \in L^{2}(\omega)$ via a wavelet basis and then analyze the behavior of the matrix of $T$ relative to this basis. One then arrives at testing conditions related to the number of moments that the basis possess, as well as a relaxation of the energy condition required to control certain terms in the matrix. With this new basis we study a $T p$ type result in the two weight setting. See Conjecture 16 below. 


\section{Weighted HAAR BASES}

In this section we review the existence, uniqueness and degeneracy of the weighted Haar wavelets, beginning with the local case. Let $\mu$ be a locally finite positive Borel measure on the unit interval $[0,1)$.

Definition 4. Set $I_{0}^{0} \equiv[0,1), I_{0}^{1} \equiv\left[0, \frac{1}{2}\right), I_{1}^{1} \equiv\left[\frac{1}{2}, 1\right)$, and in general

$$
I_{j}^{m} \equiv\left[\frac{j}{2^{m}}, \frac{j+1}{2^{m}}\right), \quad 0 \leq j \leq 2^{m}-1, m \in \mathbb{Z}_{+} .
$$

Note that the left half of the interval $I_{j}^{m}$ is $I_{j \text {, left }}^{m}=I_{2 j}^{m+1}$ and that the right half of the interval $I_{j}^{m}$ is $I_{j \text {,right }}^{m}=I_{2 j+1}^{m+1}$. We begin by briefly reviewing the weighted Haar wavelet bases on the real line and in Euclidean space, and in the next section we will turn to weighted Alpert wavelet bases with more vanishing moments.

Suppose that $\mu$ is a finite positive Borel measure on $[0,1)$ and define

$$
L^{2}(\mu) \equiv\left\{f:[0,1) \rightarrow \mathbb{C} \mu \text {-measurable }: \int|f|^{2} d \mu<\infty\right\} .
$$

Let $|E|_{\mu}$ denote the $\mu$-measure of a Borel set $E$. Assume first the nondegeneracy condition that $\mu$ charges every dyadic subinterval of $[0,1)$,

$$
|J|_{\mu}>0 \text { for all for all } J=I_{j}^{m}, m \in \mathbb{Z}_{+}, 0 \leq j \leq 2^{m}-1 .
$$

Definition 5. For all $m \in \mathbb{Z}_{+}$and $0 \leq j \leq 2^{m}-1$, define

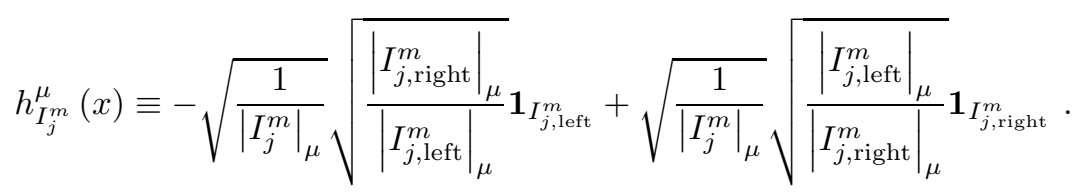

Finally define

$$
h_{0}^{\mu}(x) \equiv{\sqrt{\frac{1}{|[0,1)|_{\mu}}}}_{\mathbf{1}_{[0,1)}(x)} .
$$

Theorem 6. The collection

$$
\mathcal{U}_{[0,1)}^{\mathrm{Haar}, \mu} \equiv\left\{h_{0}^{\mu}\right\} \cup\left\{h_{I_{j}^{m}}^{\mu}\right\}_{m \in \mathbb{Z}_{+} \text {and } 0 \leq j \leq 2^{m}-1}
$$

is an orthonormal basis for $L^{2}(\mu)$.

Proof. It is a straightforward computation to see that $\mathcal{U}_{[0,1)}^{\text {Haar, }, \mu}$ is an orthonormal set in $L^{2}(\mu)$, and the dyadic Lebesgue differentiation theorem with respect to the measure $\mu$, together with the multi-resolution telescoping identities, show that $\mathcal{U}_{[0,1)}^{\mathrm{Haar}, \mu}$ is complete in $L^{2}(\mu)$.

2.1. Derivation of the explicit formula (2.2) . The coefficients on the functions $\mathbf{1}_{I_{j, \text { left }}^{m}}$ and $\mathbf{1}_{I_{j, \mathrm{right}}^{m}}$ are derived in the following way. If we set

$$
h_{I_{j}^{m}}(x) \equiv-\alpha \mathbf{1}_{I_{j, \text { left }}^{m}}+\beta I_{j, \text { right }}^{m},
$$

and demand that both

$$
\begin{aligned}
0 & =\int_{I_{j}^{m}} h_{I_{j}^{m}} d \mu=-\alpha\left|I_{j, \text { left }}^{m}\right|_{\mu}+\beta\left|I_{j, \text { right }}^{m}\right|_{\mu}, \\
1 & =\int_{I_{j}^{m}}\left|h_{I_{j}^{m}}\right|^{2} d \mu=\alpha^{2}\left|I_{j, \text { left }}^{m}\right|_{\mu}+\beta^{2}\left|I_{j, \text { right }}^{m}\right|_{\mu},
\end{aligned}
$$

then we must have

$$
\beta=\frac{\left|I_{j, \text { left }}^{m}\right|_{\mu}}{\left|I_{j, \text { right }}^{m}\right|_{\mu}} \alpha \text { and } 1=\alpha^{2}\left|I_{j, \text { left }}^{m}\right|_{\mu}+\left(\frac{\left|I_{j, \text { left }}^{m}\right|_{\mu}}{\left|I_{j, \text { right }}^{m}\right|_{\mu}}\right)^{2} \alpha^{2}\left|I_{j, \text { right }}^{m}\right|_{\mu}=\alpha^{2}\left|I_{j}^{m}\right|_{\mu} \frac{\left|I_{j, \text { left }}^{m}\right|_{\mu}}{\left|I_{j, \text { right }}^{m}\right|_{\mu}}
$$


which implies

$$
\alpha=\sqrt{\frac{\left|I_{j, \text { right }}^{m}\right|_{\mu}}{\left|I_{j}^{m}\right|_{\mu}\left|I_{j, \text { left }}^{m}\right|_{\mu}}} \text { and } \beta=\sqrt{\frac{\left|I_{j, \text { left }}^{m}\right|_{\mu}}{\left|I_{j}^{m}\right|_{\mu}\left|I_{j, \text { right }}^{m}\right|_{\mu}}}
$$

where we see that $\alpha$ and $\beta$ are uniquely determined up to sign.

2.2. The Haar degenerate case. Here we examine what happens when the nondegeneracy condition (2.1) fails.

Lemma 7. If at least one of the two children of $I_{j}^{m}$ fails to be charged by $\mu$, then if $h_{I_{j}^{m}}$ is defined according to the derivation outlined above, we have that $h_{I_{j}^{m}} \equiv 0$.

Proof. Fix $I_{j}^{m}$ with $m \in \mathbb{Z}_{+}$and $0 \leq j \leq 2^{m}-1$. If $\left|I_{j}^{m}\right|_{\mu}=0$, then clearly $h_{I_{j}^{m}}=0$ in $L^{2}(\mu)$. If just one of the children of $I_{j}^{m}$ is not charged by $\mu$, say $\left|I_{j, \text { left }}^{m}\right|_{\mu}=0$ but $\left|I_{j, \text { right }}^{m}\right|_{\mu}>0$, then the moment requirement above becomes $0=-\alpha\left|I_{j, \text { left }}^{m}\right|_{\mu}+\beta\left|I_{j, \text { right }}^{m}\right|_{\mu}=\beta\left|I_{j, \text { right }}^{m}\right|_{\mu}$, which implies $\beta=0$, and hence $h_{I_{j}^{m}}=-\alpha \mathbf{1}_{I_{j, \text { left }}^{m}}+\beta I_{j, \text { right }}^{m}=-\alpha \mathbf{1}_{I_{j, \text { left }}^{m}} \equiv 0$ in $L^{2}(\mu)$. Thus we see that $h_{I_{j}^{m}} \equiv 0$ if at least one of its children is not charged by $\mu$.

The resulting pared collection $\mathcal{U}_{[0,1)}^{\mathrm{Haar}, \mu} \equiv\left\{h_{0}^{\mu}\right\} \cup\left\{h_{I_{j}^{m}}^{\mu}\right\}_{m \in \mathbb{Z}_{+} \text {and } 0 \leq j \leq 2^{m}-1}$, where now $h_{I_{j}^{m}}^{\mu}$ is removed if it vanishes identically, is still an orthonormal basis for $L^{2}(\mu)$. Indeed, this follows from the fact that the telescoping identities still hold, and hence Lebesgue's dyadic differentiation theorem continues to show the pared collection $\mathcal{U}_{[0,1)}^{\text {Haar, } \mu}$ is complete in $L^{2}(\mu)$.

2.3. The global case. If $\mu$ is a locally finite positive Borel measure on the real line $\mathbb{R}, \mathcal{D}$ is a dyadic grid in $\mathbb{R}$, and

$$
h_{I}^{\mu}(x) \equiv\left\{\begin{array}{ccc}
-\sqrt{\frac{1}{|I|_{\mu}}} \sqrt{\frac{\left|I_{\mathrm{right}}\right|_{\mu}}{\left|I_{\text {left }}\right|_{\mu}}} \mathbf{1}_{I_{\text {left }}}+\sqrt{\frac{1}{|I|_{\mu}}} \sqrt{\frac{\left|I_{\text {left }}\right|_{\mu}}{\left|I_{\text {right }}\right|_{\mu}}} \mathbf{1}_{I_{\text {right }}} & \text { if } & \min \left\{\left|I_{\text {right }}\right|_{\mu},\left|I_{\text {left }}\right|_{\mu}\right\}>0 \\
0 & \text { if } & \min \left\{\left|I_{\text {right }}\right|_{\mu},\left|I_{\text {left }}\right|_{\mu}\right\}=0
\end{array}\right.
$$

for each dyadic interval $I \in \mathcal{D}$, then the collection

$$
\mathcal{U}_{\mathbb{R}}^{\text {Haar }, \mu} \equiv\left\{h_{I}^{\mu}\right\}_{I \in \mathcal{D}}
$$

is an orthonormal basis for $L^{2}(\mu)$ (where we of course discard those $h_{I}^{\mu}$ that vanish identically).

2.4. The higher dimensional case. Again the local case $L^{2}\left([0,1)^{n} ; \mu\right)$ and the global case $L^{2}\left(\mathbb{R}^{n} ; \mu\right)$ are treated similarly and we only consider the global case $L^{2}(\mu)=L^{2}\left(\mathbb{R}^{n} ; \mu\right)$ here. So suppose that $\mu$ is a locally finite positive Borel measure on $\mathbb{R}^{n}$ and that $\mathcal{D}$ is a dyadic grid on $\mathbb{R}^{n}$. Given a dyadic cube $Q \in \mathcal{D}$ with $|Q|_{\mu}>0$, let $\triangle_{Q}^{\mu}$ denote orthogonal projection onto the finite dimensional subspace $L_{Q ; 1}^{2}(\mu)$ of $L^{2}(\mu)$ that consists of linear combinations of the indicators of the children $\mathfrak{C}(Q)$ of $Q$ that have $\mu$-mean zero over $Q$ :

$L_{Q ; 1}^{2}(\mu) \equiv\left\{f=\sum_{Q^{\prime} \in \mathfrak{C}(Q)} a_{Q^{\prime}} \mathbf{1}_{Q^{\prime}}: a_{Q^{\prime}} \in \mathbb{R}, \int_{Q} f d \mu=0\right\}=\operatorname{Span}\left\{\mathbf{1}_{Q^{\prime}}: Q^{\prime} \in \mathfrak{C}(Q),\left|Q^{\prime}\right|_{\mu}>0\right\} \ominus \operatorname{Span}\left\{\mathbf{1}_{Q}\right\}$

Thus, among other things, we see that $\operatorname{dim} L_{Q ; 1}^{2}=\#\left\{Q^{\prime} \in \mathfrak{C}(Q):\left|Q^{\prime}\right|_{\mu}>0\right\}-1$. If $|Q|_{\mu}=0$, set $\triangle_{Q}^{\mu}=0$. Then we have the important telescoping property for dyadic cubes $Q_{1} \subset Q_{2}$ (below $\left[Q_{1}, Q_{2}\right]=\left\{Q: Q_{1} \subset\right.$ $\left.\left.Q \subset Q_{2}\right\}\right)$ :

$$
\mathbf{1}_{Q_{0}}(x)\left(\sum_{Q \in\left[Q_{1}, Q_{2}\right]} \triangle_{Q}^{\mu} f(x)\right)=\mathbf{1}_{Q_{0}}(x)\left(\mathbb{E}_{Q_{0}}^{\mu} f-\mathbb{E}_{Q_{2}}^{\mu} f\right), \quad Q_{0} \in \mathfrak{C}\left(Q_{1}\right), f \in L^{2}(\mu) .
$$


We will at times find it convenient to use a fixed orthonormal basis $\left\{h_{Q}^{\mu, a}\right\}_{a \in \Gamma_{n}}$ of $L_{Q}^{2}(\mu)$ where $\Gamma_{n}$ is any convenient index set with cardinality equal to the dimension of $L_{Q}^{2}(\mu)$, i.e.

$$
\# \Gamma_{n}=\operatorname{dim} L_{Q}^{2}(\mu)=\#\left\{Q^{\prime} \in \mathfrak{C}(Q):\left|Q^{\prime}\right|_{\mu}>0\right\}-1,
$$

where the second equality here follows from the fact that the functions in $L_{Q}^{2}(\mu)$ have vanishing mean. Then $\left\{h_{Q}^{\mu, a}\right\}_{a \in \Gamma_{n} \text { and } Q \in \mathcal{D}}$ is an orthonormal basis for $L^{2}(\mu)$, with the understanding that we add the constant function 1 if $\mu$ is a finite measure. In particular, if $\mu$ is an infinite measure, we have

$$
\begin{aligned}
f(x) & =\sum_{Q \in \mathcal{D}} \triangle_{Q}^{\mu} f(x), \quad \mu-\text { a.e. } x \in \mathbb{R}^{n}, \\
\|f\|_{L^{2}(\mu)}^{2} & =\sum_{Q \in \mathcal{D}}\left\|\triangle_{Q}^{\mu} f\right\|_{L^{2}(\mu)}^{2}=\sum_{Q \in \mathcal{D}} \sum_{a \in \Gamma_{n}}|\widehat{f}(Q)|^{2},
\end{aligned}
$$

where

$$
|\widehat{f}(Q)|^{2} \equiv \sum_{a \in \Gamma_{n}}\left|\left\langle f, h_{Q}^{\mu, a}\right\rangle_{\mu}\right|^{2}
$$

and the measure is suppressed in the notation. Indeed, this follows from (2.3) and Lebesgue's differentiation theorem for cubes.

\section{Weighted Alpert Wavelets With higher Vanishing moments}

Let $\mu$ be a locally finite positive Borel measure on $\mathbb{R}^{n}$, and fix $k \in \mathbb{N}$. In analogy with the definition of $L_{Q ; 1}^{2}(\mu)$ above for $Q \in \mathcal{P}^{n}$, we denote by $L_{Q ; k}^{2}(\mu)$ the finite dimensional subspace of $L^{2}(\mu)$ that consists of linear combinations of the indicators of the children $\mathfrak{C}(Q)$ of $Q$ multiplied by polynomials of degree at most $k-1$, and such that the linear combinations have vanishing $\mu$-moments on the cube $Q$ up to order $k-1$ :

$$
L_{Q ; k}^{2}(\mu) \equiv\left\{f=\sum_{Q^{\prime} \in \mathfrak{C}(Q)} \mathbf{1}_{Q^{\prime}} p_{Q^{\prime} ; k}(x): \int_{Q} f(x) x_{i}^{\ell} d \mu(x)=0, \quad \text { for } 0 \leq \ell \leq k-1 \text { and } 1 \leq i \leq n\right\},
$$

where $p_{Q^{\prime} ; k}(x)=\sum_{\alpha \in \mathbb{Z}_{+}^{n}:|\alpha| \leq k-1} \quad a_{Q^{\prime} ; \alpha} x^{\alpha}$ is a polynomial in $\mathbb{R}^{n}$ of degree $|\alpha|=\alpha_{1}+\ldots+\alpha_{n}$ at most $k-1$. Here $x^{\alpha}=x_{1}^{\alpha_{1}} x_{2}^{\alpha_{2}} \ldots x_{n}^{\alpha_{n}}$. Let $d_{Q ; k} \equiv \operatorname{dim} L_{Q ; k}^{2}(\mu)$ be the dimension of the finite dimensional linear space $L_{Q ; k}^{2}(\mu)$. Note that the space $L_{Q ; k}^{2}(\mu)$ can also be written as:

$$
\operatorname{Span}\left\{\mathbf{1}_{Q^{\prime}} x^{\alpha}: Q^{\prime} \in \mathfrak{C}(Q),\left|Q^{\prime}\right|_{\mu}>0,|\alpha| \leq k-1\right\} \ominus\left(\oplus_{\alpha:|\alpha| \leq k-1} \mathbf{1}_{Q} x^{\alpha}\right) .
$$

We begin with the proof of Theorem 1 in the next subsection below, and we will complete the proof of Theorem 2 in the third subsection. In the final subsection we will give a complete and detailed answer to both uniqueness and degeneracy in the special case when $n=1$ and $k=2$.

3.1. Proof of Theorem 1, We begin with an estimate of the dimension $d_{Q ; k}$.

Proposition 8. We have:

$$
0 \leq d_{Q ; k} \leq\left(2^{n}-1\right) A_{k, n}=\left(2^{n}-1\right)\left(\begin{array}{c}
n+k-1 \\
n
\end{array}\right),
$$

where $A_{k, n}$ denotes the number of non-negative integer solutions to $\alpha_{1}+\cdots+\alpha_{n} \leq k-1$.

Proof. First, if the functions $\left\{\mathbf{1}_{Q}(x) x^{\alpha}:|\alpha| \leq k-1\right\}$ are linearly independent, then

$$
\operatorname{dim} \operatorname{Span}\left\{\mathbf{1}_{Q}(x) x^{\alpha}:|\alpha| \leq k-1\right\}=A_{k, n} .
$$

Now, let $B$ denote $\operatorname{dim} \operatorname{Span}\left\{\mathbf{1}_{Q}(x) x^{\alpha}:|\alpha| \leq k-1\right\}$. Then it follows that

$$
\operatorname{dim} \operatorname{Span}\left\{\mathbf{1}_{Q^{\prime}}(x) x^{\alpha}:|\alpha| \leq k-1\right\} \leq B, \quad \text { for all } Q^{\prime} \in \mathfrak{C}(Q) .
$$

Thus

$$
\operatorname{dim} L_{Q ; k}^{2}(\mu) \leq \#\left\{Q^{\prime} \in \mathfrak{C} Q:\left|Q^{\prime}\right|_{\mu}>0\right\} B-B \leq\left(2^{n}-1\right) B \leq\left(2^{n}-1\right) A_{k, n} .
$$


Finally it is well-known that the number of nonnegative solutions to $\alpha_{1}+\cdots+\alpha_{n}=j$ is $\left(\begin{array}{c}n-1+j \\ j\end{array}\right)$ ( choose $n-1$ boxes from a row of $n+j-1$ boxes and put a single ball in each of the unchosen boxes - then let $\alpha_{i}$ be the number of balls between the $(i-1)^{t h}$ box and the $i^{t h}$ box $)$, and so $A_{k, n}=\sum_{j=0}^{k-1}\left(\begin{array}{c}n-1+j \\ j\end{array}\right)=\left(\begin{array}{c}n-1+k \\ k-1\end{array}\right)$ by induction on $k$.

Now we restrict attention to a fixed dyadic grid $\mathcal{D}$ in $\mathbb{R}^{n}$. For $P, Q \in \mathcal{D}$ dyadic cubes, the subspaces $L_{P ; k}^{2}(\mu)$ and $L_{Q ; k}^{2}(\mu)$ are orthogonal for $P \neq Q$, i.e. $\langle f, g\rangle_{L^{2}(\mu)}=0$ for $f \in L_{P ; k}^{2}(\mu)$ and $g \in L_{Q ; k}^{2}(\mu)$. Indeed, the only case that needs checking is when either $P \varsubsetneqq Q$ or $Q \varsubsetneqq P$. If $P \subsetneq Q$, then the restriction of $g$ to $P$ is a polynomial of degree less than or equal to $k-1$. But by definition, $L_{P ; k}^{2}(\mu)$ is orthogonal to such functions and so $\langle f, g\rangle_{L^{2}(\mu)}=0$. Of course similar reasoning holds in the case $Q \subsetneq P$.

Now define

$$
\begin{gathered}
\mathcal{F}_{\infty}^{k}(\mu) \equiv\left\{\alpha \in \mathbb{Z}_{+}^{n}:|\alpha| \leq k-1: x^{\alpha} \in L^{2}(\mu)\right\}, \\
\text { and } \mathcal{P}_{\mathbb{R}^{n}}^{k}(\mu) \equiv \operatorname{Span}\left\{x^{\alpha}\right\}_{\alpha \in \mathcal{F}_{\infty}^{k}} .
\end{gathered}
$$

We claim that $L^{2}(\mu)$ is the Hilbert space direct sum of $\mathcal{P}_{\mathbb{R}^{n}}^{k}(\mu)$ in $L^{2}(\mu)$ and the finite dimensional subspaces $\left\{L_{Q ; k}^{2}(\mu)\right\}_{Q \in \mathcal{D}}$, namely

$$
L^{2}(\mu)=\mathcal{P}_{\mathbb{R}^{n}}^{k}(\mu) \oplus\left(\oplus_{Q \in \mathcal{D}} L_{Q ; k}^{2}(\mu)\right) .
$$

To see this, fix a large dyadic cube $P \in \mathcal{D}$. Set

$$
\mathcal{P}_{P}^{k} \equiv\left\{f=\mathbf{1}_{P}(x) p_{k}(x): \alpha \in \mathbb{Z}_{+}^{n}:|\alpha| \leq k-1\right\}
$$

to be the linear space of restrictions to $P$ of polynomials $p_{k}(x)$ having degree at most $k-1$. Then for $Q \subset P$, we have

$$
\begin{aligned}
L_{Q ; 1}^{2}(\mu) & =\left\{f=\sum_{Q^{\prime} \in \mathfrak{C}(Q)} a_{Q^{\prime}} \mathbf{1}_{Q^{\prime}}: a_{Q^{\prime}} \in \mathbb{R}, \int_{Q} f d \mu=0\right\} \\
& \subset \overline{\operatorname{Span}}\left\{\mathcal{P}_{P}^{k},\left\{L_{R ; k}^{2}(\mu)\right\}_{R \in \mathcal{D}: Q \subset R \subset P}\right\} .
\end{aligned}
$$

Now let $P$ tend to infinity to conclude that

$$
L_{Q ; 1}^{2}(\mu) \subset \overline{\operatorname{Span}}\left\{\mathcal{P}_{\mathbb{R}^{n}}^{k}(\mu),\left\{L_{R ; k}^{2}(\mu)\right\}_{R \in \mathcal{D}: Q \subset R}\right\} .
$$

But we already know that the Haar spaces $\mathcal{P}_{\mathbb{R}^{n}}^{1}(\mu)$ and $L_{Q ; 1}^{2}(\mu)$ form a direct sum decomposition of $L^{2}(\mu)$, i.e.

$$
L^{2}(\mu)=\mathcal{P}_{\mathbb{R}^{n}}^{1}(\mu) \oplus\left(\oplus_{Q \in \mathcal{D}} L_{Q ; 1}^{2}(\mu)\right),
$$

and hence we see that

$$
L^{2}(\mu)=\mathcal{P}_{\mathbb{R}^{n}}^{1}(\mu) \oplus\left(\oplus_{Q \in \mathcal{D}} L_{Q ; 1}^{2}(\mu)\right) \subset \mathcal{P}_{\mathbb{R}^{n}}^{k}(\mu) \oplus\left(\oplus_{Q \in \mathcal{D}} L_{Q ; k}^{2}(\mu)\right) \subset L^{2}(\mu) .
$$

Formula (3.1) gives the telescoping identities (1.1), and the moment conditions (1.2) are immediate from the definition of $L_{Q ; k}^{2}(\mu)$. This completes the proof of Theorem 1

3.2. Uniqueness and degeneracy. In this subsection we begin to investigate the lack of uniqueness of the orthogonal projections $\left\{\triangle_{\mathbb{R}^{n} ; k}^{\mu}\right\} \cup\left\{\triangle_{Q ; k}^{\mu}\right\}_{Q \in \mathcal{D}^{n}}$ and their degeneracy when

$$
d_{Q ; k}<\left(2^{n}-1\right)\left(\begin{array}{c}
n+k-1 \\
n
\end{array}\right)
$$

is not maximal. We can of course use the Gram-Schmidt orthogonalization algorithm to find an orthonormal basis $\left\{k_{\mathbb{R}^{n}}^{\mu, a}\right\}_{a \in \Gamma_{n}^{k}\left(\mathbb{R}^{n}\right)}$ of $\mathcal{P}_{\mathbb{R}^{n}}^{k}(\mu)$ where $\Gamma_{n}^{k}\left(\mathbb{R}^{n}\right)$ is any convenient index set with cardinality equal to $\operatorname{dim} \mathcal{P}_{\mathbb{R}^{n}}^{k}(\mu)=\# \mathcal{F}_{\infty}^{k}(\mu)$, and also an orthonormal basis $\left\{h_{Q}^{\mu, a}\right\}_{a \in \Gamma_{n}^{k}}$ of $L_{Q ; k}^{2}(\mu)$ where $\Gamma_{n}^{k}$ is any convenient index set with cardinality equal to $d_{Q ; k}$, the dimension of $L_{Q}^{2,1}(\mu)$, i.e.

$$
\# \Gamma_{n}^{k}(Q)=\operatorname{dim} L_{Q ; k}^{2}(\mu) .
$$


Then for any $J \in \mathcal{D} \cup\left\{\mathbb{R}^{n}\right\}$, the set $\left\{k_{J}^{\mu, a}\right\}_{a \in \Gamma_{n}^{k}(J)} \cup\left\{h_{Q}^{\mu, a}\right\}_{a \in \Gamma_{n}^{k}(Q) \text { and } Q \in \mathcal{D} \text { with } Q \subset J}$ is an orthonormal basis for $L^{2}\left(\mathbf{1}_{J} \mu\right)$.

In the case of dimension $n=1$ with $k=1$ vanishing moment, Proposition 8 shows that the dimension $d_{Q ; 1}$ of the subspace $L_{Q ; 1}^{2}(\mu)$ satisfies $0 \leq d_{Q ; 1} \leq\left(2^{1}-1\right)\left(\begin{array}{c}1+1-1 \\ 1-1\end{array}\right)=1$. So if $L_{Q ; 1}^{2}(\mu) \neq\{0\}$, the orthogonal projection $\triangle_{Q ; 1}^{\mu}$ is one-dimensional, and hence is given by $\triangle_{Q ; 1}^{\mu} f=\frac{\left\langle f, h_{Q}^{\mu}\right\rangle}{\left\langle h_{Q}^{\mu}, h_{Q}^{\mu}\right\rangle} h_{Q}^{\mu}$ for a function $h_{Q}^{\mu}$, which has the especially simple formula given by (2.2). We next investigate to what extent one can find "nice" explicit bases of $L_{Q ; k}^{2}(\mu)$ in dimension $n=1$, where in this case $0 \leq d_{Q ; k} \leq\left(2^{1}-1\right)\left(\begin{array}{c}1+k-1 \\ k-1\end{array}\right)=k$.

3.3. Proof of Theorem 2, To prepare for the proof of Theorem 2, we first turn to the explicit construction of weighted Alpert bases in dimension $n=1$ when the number of vanishing moments is $k \geq 2$.

3.3.1. An explicit basis for $n=1$ and $k \geq 2$. Let $\mu$ be a locally finite positive Borel measure on $\mathbb{R}$ that satisfies the Alpert nondegeneracy condition

$$
\boldsymbol{M}_{J, k} \equiv\left[\begin{array}{ccccc}
\left|J^{(0)}\right|_{\mu} & \left|J^{(1)}\right|_{\mu} & \cdots & \left|J^{(k-2)}\right|_{\mu} & \left|J^{(k-1)}\right|_{\mu} \\
\left|J^{(1)}\right|_{\mu} & \left|J^{(2)}\right|_{\mu} & \ddots & \left|J^{(k-1)}\right|_{\mu} & \left|J^{(k)}\right|_{\mu} \\
\vdots & \ddots & \ddots & \ddots & \vdots \\
\left|J^{(k-2)}\right|_{\mu} & \left|J^{(k-1)}\right|_{\mu} & \ddots & \left|J^{(2 k-4)}\right|_{\mu} & \left|J^{(2 k-3)}\right|_{\mu} \\
\left|J^{(k-1)}\right|_{\mu} & \left|J^{(k)}\right|_{\mu} & \cdots & \left|J^{(2 k-3)}\right|_{\mu} & \left|J^{(2 k-2)}\right|_{\mu}
\end{array}\right] \succ 0
$$

for all $J \in \mathcal{D}$, and where we denote a positive definite matrix $A$ by $A \succ 0$. For each $x \in J$ we have that

$$
\boldsymbol{M}(x) \equiv\left[\begin{array}{ccccc}
1 & x & \cdots & x^{k-2} & x^{k-1} \\
x & x^{2} & \ddots & x^{k-3} & x^{k-2} \\
\vdots & \ddots & \ddots & \ddots & \vdots \\
x^{k-2} & x^{k-3} & \ddots & x^{2 k-4} & x^{2 k-3} \\
x^{k-1} & x^{k-2} & \cdots & x^{2 k-3} & x^{2 k-2}
\end{array}\right]=\left[\begin{array}{c}
1 \\
x \\
\vdots \\
x^{k-2} \\
x^{k-1}
\end{array}\right]\left[\begin{array}{lllll}
1 & x & \cdots & x^{k-2} & x^{k-1}
\end{array}\right]
$$

is a dyad, namely a rank one nonnegative semidefinite matrix. Thus

$$
\boldsymbol{M}_{J, k}=\int_{J}\left[\begin{array}{ccccc}
1 & x & \cdots & x^{k-2} & x^{k-1} \\
x & x^{2} & \ddots & x^{k-3} & x^{k-2} \\
\vdots & \ddots & \ddots & \ddots & \vdots \\
x^{k-2} & x^{k-3} & \ddots & x^{2 k-4} & x^{2 k-3} \\
x^{k-1} & x^{k-2} & \cdots & x^{2 k-3} & x^{2 k-2}
\end{array}\right] d \mu(x)=\int_{J} \boldsymbol{M}(x) d \mu(x)
$$

is also a nonnegative semidefinite matrix.

We now claim that the matrix $\boldsymbol{M}_{J, k}$ is positive definite if and only if the functions

$$
\left\{\mathbf{1}_{J}(x), x \mathbf{1}_{J}(x), \ldots, x^{k-1} \mathbf{1}_{J}(x)\right\}
$$

are linearly independent in $L^{2}(\mu)$. Indeed, in the special case $k=2$ we have

$$
\operatorname{det} \boldsymbol{M}_{J, 2}=\operatorname{det}\left[\begin{array}{ll}
\left|J^{(0)}\right|_{\mu} & \left|J^{(1)}\right|_{\mu} \\
\left|J^{(1)}\right|_{\mu} & \left|J^{(2)}\right|_{\mu}
\end{array}\right]>0
$$

if and only if

$$
\left|J^{(1)}\right|_{\mu}^{2}=\left(\int_{J} x d \mu(x)\right)^{2} \leq\left(\int_{J} 1^{2} d \mu(x)\right)\left(\int_{J} x^{2} d \mu(x)\right)=\left|J^{(0)}\right|_{\mu}\left|J^{(2)}\right|_{\mu},
$$

with strict inequality if and only if the functions $\mathbf{1}_{J}(x)$ and $x \mathbf{1}_{J}(x)$ are linearly independent in $L^{2}(\mu)$. Here is the general case. 
Theorem 9. Let $\mu$ be a locally finite positive Borel measure on $\mathbb{R}$. Then:

(1) the $k \times k$ matrix of moments

$$
\boldsymbol{M}_{J, k}=\int\left[\begin{array}{ccccc}
1 & x & \cdots & x^{k-2} & x^{k-1} \\
x & x^{2} & \ddots & x^{k-3} & x^{k-2} \\
\vdots & \ddots & \ddots & \ddots & \vdots \\
x^{k-2} & x^{k-3} & \ddots & x^{2 k-4} & x^{2 k-3} \\
x^{k-1} & x^{k-2} & \cdots & x^{2 k-3} & x^{2 k-2}
\end{array}\right] d \mu(x)
$$

is nonnegative semidefinite, and

(2) $\boldsymbol{M}_{J, k}$ has rank $\ell$ if and only if the span of the functions $1, x, x^{2}, . ., x^{k-1}$ has dimension $\ell$ in $L^{2}(\mu)$, i.e.

$$
\operatorname{rank} \boldsymbol{M}_{J, k}=\operatorname{dim} \operatorname{Span}\left\{x^{j}\right\}_{j=0}^{k-1} .
$$

Proof. With $V_{k}(x)^{\operatorname{tr}} \equiv\left[\begin{array}{lllll}1 & x & \cdots & x^{k-2} & x^{k-1}\end{array}\right]$, we compute

$$
\left[\begin{array}{ccccc}
1 & x & \cdots & x^{k-2} & x^{k-1} \\
x & x^{2} & \ddots & x^{k-3} & x^{k-2} \\
\vdots & \ddots & \ddots & \ddots & \vdots \\
x^{k-2} & x^{k-3} & \ddots & x^{2 k-4} & x^{2 k-3} \\
x^{k-1} & x^{k-2} & \cdots & x^{2 k-3} & x^{2 k-2}
\end{array}\right]=\left[\begin{array}{c}
1 \\
x \\
\vdots \\
x^{k-2} \\
x^{k-1}
\end{array}\right]\left[\begin{array}{lllll}
1 & x & \cdots & x^{k-2} & x^{k-1}
\end{array}\right]=V_{k}(x) V_{k}(x)^{\operatorname{tr}},
$$

and so

$$
\boldsymbol{M}_{J, k} \equiv \int_{\mathbb{R}^{k}} V_{k}(x) V_{k}(x)^{\operatorname{tr}} d \mu(x)
$$

is a positive integral of dyads, hence nonnegative semidefinite. Now note that the quadratic form

$$
\xi^{\operatorname{tr}} \boldsymbol{M}_{J, k} \xi=\int_{\mathbb{R}^{k}}\left(\xi^{\operatorname{tr}} V_{k}(x)\right)\left(V_{k}(x)^{\operatorname{tr}} \xi\right) d \mu(x)=\int_{\mathbb{R}^{k}}\left(\xi \cdot V_{k}(x)\right)^{2} d \mu(x)
$$

is strictly positive if and only if $\operatorname{Span}\left\{V_{k}(x): x \in \operatorname{supp} \mu\right\}$ has dimension $k$. Indeed, $\operatorname{dim} \operatorname{Span}\left\{V_{k}(x): x \in \operatorname{supp} \mu\right\}=$ $k$ if and only if the function $\xi \cdot V_{k}(x)$ is not trivial for every $\xi \in \mathbb{R}^{k} \backslash\{0\}$, and this in turn happens if and only if $\xi^{\mathrm{tr}} \boldsymbol{M}_{J, k} \xi>0$ for all $\xi \in \mathbb{R}^{k} \backslash\{0\}$. Similarly we have $\operatorname{dim} \operatorname{Span}\left\{V_{k}(x): x \in \operatorname{supp} \mu\right\}=\ell$ if and only if the vector subspace of $\mathbb{R}^{k}$ defined by

$$
S \equiv\left\{\xi \in \mathbb{R}^{k}: \xi \cdot V_{k}(x) \text { is not trivial }\right\}
$$

has dimension $\ell$, and this in turn happens if and only if $\operatorname{dim} \operatorname{Span}\left\{x^{j}\right\}_{j=0}^{k-1}=\ell$.

Remark 10. The van der Monde determinant formula shows that $\operatorname{dim} \operatorname{Span}\left\{x^{j}\right\}_{j=0}^{k-1}=\ell<k$ if and only if the measure $\mu$ is a sum of positive multiples of $\ell$ point masses, i.e. $\mu=\sum_{j=1}^{\ell} c_{j} \delta_{x_{j}}$ with $c_{j}>0$ and the $x_{j}$ distinct.

3.3.2. An orthonormal basis of the multiresolution projections. Let $\mu$ be a locally finite positive Borel measure on $\mathbb{R}$ that satisfies the Alpert nondegeneracy condition (3.2), i.e. the determinant of all principal $\ell \times \ell$ submatrices $\boldsymbol{M}_{J, \mathbf{i}}$ is positive for $\mathbf{i}=\left(i_{1}, i_{2}, \ldots, i_{\ell}\right)$ and $1 \leq \ell \leq k$,

$\operatorname{det} \boldsymbol{M}_{J, \mathbf{i}}>0, \quad$ for all $1 \leq \ell \leq k$, and all $J=I_{j}^{m}, m \in \mathbb{Z}_{+}, 0 \leq j \leq 2^{m}-1$;

where $\boldsymbol{M}_{J, \mathbf{i}} \equiv\left[\begin{array}{ccccc}\left|J^{\left(2 i_{1}\right)}\right|_{\mu} & \left|J^{\left(i_{1}+i_{2}\right)}\right|_{\mu} & \cdots & \left|J^{\left(i_{1}+i_{\ell-1}\right)}\right|_{\mu} & \left|J^{\left(i_{1}+i_{\ell}\right)}\right|_{\mu} \\ \left|J^{\left(i_{2}+i_{1}\right)}\right|_{\mu} & \left|J^{\left(2 i_{2}\right)}\right|_{\mu} & \ddots & \left|J^{\left(i_{2}+i_{\ell-1}\right)}\right|_{\mu} & \left|J^{\left(i_{2}+i_{\ell}\right)}\right|_{\mu} \\ \vdots & \ddots & \ddots & \ddots & \vdots \\ \left|J^{\left(i_{\ell-1}+i_{1}\right)}\right|_{\mu} & \left|J^{\left(i_{\ell-1}+i_{2}\right)}\right|_{\mu} & \ddots & \left|J^{\left(2 i_{\ell-1}\right)}\right|_{\mu} & \left|J^{\left(i_{\ell-1}+i_{\ell}\right)}\right|_{\mu} \\ \left|J^{\left(i_{\ell}+i_{1}\right)}\right|_{\mu} & \left|J^{\left(i_{\ell}+i_{2}\right)}\right|_{\mu} & \cdots & \left|J^{\left(i_{\ell}+i_{\ell-1}\right)}\right|_{\mu} & \left|J^{\left(2 i_{\ell}\right)}\right|_{\mu}\end{array}\right]$. 
If for $\mathbf{i}=\left(i_{1}, i_{2}, \ldots, i_{\ell}\right)$ we set $V_{\mathbf{i}}(x)^{\operatorname{tr}} \equiv\left[\begin{array}{lllll}x^{i_{1}} & x^{i_{2}} & \cdots & x^{i_{\ell-1}} & x^{i_{\ell}}\end{array}\right]$, then by the above theorem,

$$
\boldsymbol{M}_{J, \mathbf{i}} \equiv \int_{J} V_{\mathbf{i}}(x) V_{\mathbf{i}}(x)^{\operatorname{tr}} d \mu(x)
$$

is nonnegative semidefinite; and is positive definite if and only if the the span of the functions $x^{i_{1}}, x^{i_{2}}, \ldots, x^{i_{\ell}}$ has dimension $\ell$ in $L^{2}(\mu)$, i.e.

$$
\operatorname{rank} \boldsymbol{M}_{J, \mathbf{i}}=\operatorname{dim} \operatorname{Span}\left\{x^{i_{j}}\right\}_{j=1}^{\ell} .
$$

Remark 11. Recall that a matrix $M$ is nonnegative semidefinite if and only if $\operatorname{det} \boldsymbol{M}_{\mathbf{i}} \geq 0$ for all principal submatrices $\boldsymbol{M}_{\mathbf{i}}$ (the numbers $\operatorname{det} \boldsymbol{M}_{\mathbf{i}}$ are usually referred to as principal minors); and that a matrix $M$ is positive definite if and only if $\operatorname{det} \boldsymbol{M}_{\mathbf{i}}>0$ for the special subset of leading principal submatrices $\boldsymbol{M}_{\mathbf{i}}$ having $\mathbf{i}=(1,2, \ldots, \ell)$ and $1 \leq \ell \leq k$ (in which case $\operatorname{det} \boldsymbol{M}_{\mathbf{i}}>0$ for all principal submatrices $\boldsymbol{M}_{\mathbf{i}}$ ).

Recall that we can use the Gram-Schmidt orthogonalization algorithm to find an orthonormal basis $\left\{h_{Q}^{\mu, a}\right\}_{a \in \Gamma_{1}^{k}}$ of $L_{Q ; k}^{2}(\mu)$ where $\Gamma_{1}^{k}$ is any convenient index set with cardinality equal to $d_{Q ; k}$, the dimension of $L_{Q}^{2,1}(\mu)$, i.e.

$$
\# \Gamma_{1}^{k}=\operatorname{dim} L_{Q ; k}^{2}(\mu) .
$$

Then $\left\{x^{\alpha}\right\}_{\alpha \in \mathcal{F}_{\infty}} \cup\left\{h_{Q}^{\mu, a}\right\}_{a \in \Gamma_{n}^{k} \text { and } Q \in \mathcal{D}}$ is an orthonormal basis for $L_{\mathbb{R}^{n}}^{2}(\mu)$

In the case of dimension $n=1$ with $k \geq 2$ vanishing moments, the above proposition shows that the dimension $d_{Q ; k}$ of the subspace $L_{Q ; k}^{2}(\mu)$ satisfies $0 \leq d_{Q ; k} \leq\left(2^{1}-1\right)\left(\begin{array}{c}1+k-1 \\ k-1\end{array}\right)=k$, and in the case where $\boldsymbol{M}_{J, k}$ is positive definite, we have $d_{Q ; k}=k$. Now we begin an explicit construction of an 'Alpert' orthonormal basis.

We require the Alpert functions

$$
\begin{aligned}
a_{I}^{\mu, \ell}(x) \equiv & \left(\alpha_{k-1}^{\ell} x^{k-1}+\alpha_{k-2}^{\ell} x^{k-2}+\ldots+\alpha_{1}^{\ell} x+\alpha_{0}^{\ell}\right) \mathbf{1}_{I_{\mathrm{left}}}(x) \\
& +\left(\beta_{k-1}^{\ell} x^{k-1}+\beta_{k-2}^{\ell} x^{k-2}+\ldots+\beta_{1}^{\ell} x+\beta_{0}^{\ell}\right) \mathbf{1}_{I_{\mathrm{right}}}(x),
\end{aligned}
$$

namely

$$
\begin{aligned}
a_{I}^{\mu, 1}(x) \equiv & \left(\alpha_{k-1}^{1} x^{k-1}+\alpha_{k-2}^{1} x^{k-2}+\ldots+\alpha_{1}^{1} x+\alpha_{0}^{1}\right) \mathbf{1}_{I_{\text {left }}}(x) \\
& +\left(\beta_{k-1}^{1} x^{k-1}+\beta_{k-2}^{1} x^{k-2}+\ldots+\beta_{1}^{1} x+\beta_{0}^{1}\right) \mathbf{1}_{I_{\mathrm{right}}}(x), \\
a_{I}^{\mu, 2}(x) \equiv & \left(\alpha_{k-1}^{2} x^{k-1}+\alpha_{k-2}^{2} x^{k-2}+\ldots+\alpha_{1}^{2} x+\alpha_{0}^{2}\right) \mathbf{1}_{I_{\mathrm{left}}}(x) \\
& +\left(\beta_{k-1}^{2} x^{k-1}+\beta_{k-2}^{2} x^{k-2}+\ldots+\beta_{1}^{2} x+\beta_{0}^{2}\right) \mathbf{1}_{I_{\mathrm{right}}}(x), \\
& \vdots \\
a_{I}^{\mu, k}(x) \equiv & \left(\alpha_{k-1}^{k} x^{k-1}+\alpha_{k-2}^{k} x^{k-2}+\ldots+\alpha_{1}^{k} x+\alpha_{0}^{k}\right) \mathbf{1}_{I_{\mathrm{left}}}(x) \\
& +\left(\beta_{k-1}^{k} x^{k-1}+\beta_{k-2}^{k} x^{k-2}+\ldots+\beta_{1}^{k} x+\beta_{0}^{k}\right) \mathbf{1}_{I_{\mathrm{right}}}(x),
\end{aligned}
$$

to satisfy the $k^{2}$ moment properties,

$$
\int a_{I}^{\mu, \ell}(x) x^{i} d \mu(x)=0, \quad \text { for all } 1 \leq \ell \leq k \text { and } 0 \leq i \leq k+\ell-2,
$$

the $\left(\begin{array}{c}k \\ 2\end{array}\right)$ orthogonality properties,

$$
\int a_{I}^{\mu, \ell}(x) a_{I}^{\mu, \ell^{\prime}}(x) d \mu(x)=0, \quad \text { for all } 1 \leq \ell, \ell^{\prime} \leq k,
$$

and the $k$ normalization properties,

$$
\left(\int\left|a_{I}^{\mu, \ell}(x)\right|^{2} d \mu(x)\right)^{\frac{1}{2}}=1, \quad \text { for all } 1 \leq \ell \leq k .
$$


Dimension count: Note that $k^{2}+\left(\begin{array}{c}k \\ 2\end{array}\right)+k=2 k^{2}-\left(\begin{array}{c}k \\ 2\end{array}\right)$, so that there are $\left(\begin{array}{c}k \\ 2\end{array}\right)$ degrees of

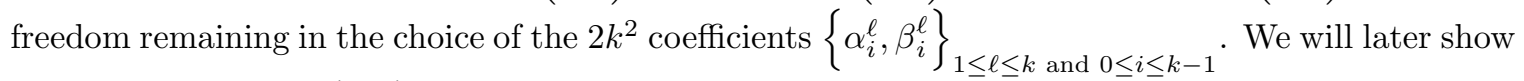
that we can impose $\left(\begin{array}{c}k \\ 2\end{array}\right)$ additional moment conditions.

So with the definition $\left|J^{(i)}\right|_{\mu} \equiv \int_{J} x^{i} d \mu(x)$, we first tackle the moment properties:

$$
\begin{aligned}
0= & \int a_{I}^{\mu, \ell}(x) x^{i} d \mu(x)=\int_{I_{\text {left }}}\left(\alpha_{k-1}^{\ell} x^{k-1}+\alpha_{k-2}^{\ell} x^{k-2}+\ldots+\alpha_{1}^{\ell} x+\alpha_{0}^{\ell}\right) x^{i} d \mu(x) \\
& +\int_{I_{\text {right }}}\left(\beta_{k-1}^{\ell} x^{k-1}+\beta_{k-2}^{\ell} x^{k-2}+\ldots+\beta_{1}^{\ell} x+\beta_{0}^{\ell}\right) x^{i} d \mu(x) \\
= & \alpha_{k-1}^{\ell}\left|I_{\text {left }}^{(i+k-1)}\right|_{\mu}+\alpha_{k-2}^{\ell}\left|I_{\text {left }}^{(i+k-2)}\right|_{\mu}+\ldots+\alpha_{0}^{\ell}\left|I_{\text {left }}^{(i)}\right|_{\mu} \\
& +\beta_{k-1}^{\ell}\left|I_{\text {right }}^{(i+k-1)}\right|_{\mu}+\beta_{k-2}^{\ell}\left|I_{\text {right }}^{(i+k-2)}\right|_{\mu}+\ldots+\beta_{0}^{\ell}\left|I_{\text {right }}^{(i)}\right|_{\mu},
\end{aligned}
$$

which lead to

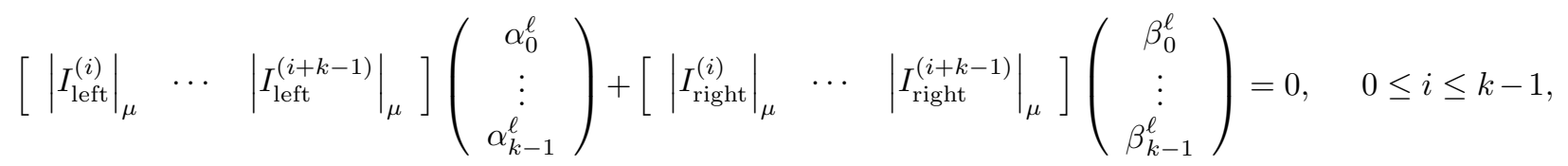

which in matrix form is,

$$
\begin{aligned}
\left(\begin{array}{c}
0 \\
0 \\
\vdots \\
0
\end{array}\right)= & {\left.\left[\begin{array}{ccccc}
\left|I_{\text {left }}^{(0)}\right|_{\mu} & \left|I_{\text {left }}^{(1)}\right|_{\mu} & \cdots & \left|I_{\text {left }}^{(k-2)}\right|_{\mu} & \left|I_{\text {left }}^{(k-1)}\right|_{\mu} \\
\left|I_{\text {left }}^{(1)}\right|_{\mu} & \left|I_{\text {left }}^{(2)}\right|_{\mu} & \cdots & \left|I_{\text {left }}^{(k-1)}\right|_{\mu} & \left|I_{\text {left }}^{(k)}\right|_{\mu} \\
\vdots & \vdots & & & \vdots \\
\left|I_{\text {left }}^{(k-2)}\right|_{\mu} & \left|I_{\text {left }}^{(k-1)}\right|_{\mu} & \ldots & \left|I_{\text {left }}^{(2 k-4)}\right|_{\mu} & \left|I_{\text {left }}^{(2 k-3)}\right|_{\mu} \\
\left|I_{\text {left }}^{(k-1)}\right|_{\mu} & \left|I_{\text {left }}^{(k)}\right|_{\mu} & \ldots & \left|I_{\text {left }}^{(2 k-3)}\right|_{\mu} & \left|I_{\text {left }}^{(2 k-2)}\right|_{\mu}
\end{array}\right] \begin{array}{c}
\alpha_{0}^{\ell} \\
\alpha_{1}^{\ell} \\
\vdots \\
\alpha_{k-2}^{\ell} \\
\alpha_{k-1}^{\ell}
\end{array}\right) } \\
& \left.+\left[\begin{array}{ccc|c|c|}
\left|I_{\text {right }}^{(0)}\right|_{\mu} & \left|I_{\text {right }}^{(1)}\right|_{\mu} & \cdots & \left|I_{\text {right }}^{(k-2)}\right|_{\mu} & \left|I_{\text {right }}^{(k-1)}\right|_{\mu} \\
\left|I_{\text {right }}^{(1)}\right|_{\mu} & \left|I_{\text {right }}^{(2)}\right|_{\mu} & \cdots & \left|I_{\text {right }}^{(k-1)}\right|_{\mu} & \left|I_{\text {right }}^{(k)}\right|_{\mu} \\
\vdots & \vdots & & & \vdots \\
\left|I_{\text {right }}^{(k-2)}\right|_{\mu} & \left|I_{\text {right }}^{(k-1)}\right|_{\mu} & \ldots & \left|I_{\text {right }}^{(2 k-4)}\right|_{\mu} & \left|I_{\text {right }}^{(2 k-3)}\right|_{\mu} \\
\left|I_{\text {right }}^{(k-1)}\right|_{\mu} & \left|I_{\text {right }}^{(k)}\right|_{\mu} & \cdots & \left|I_{\text {right }}^{(2 k-3)}\right|_{\mu} & \left|I_{\text {right }}^{(2 k-2)}\right|_{\mu}
\end{array}\right] \begin{array}{c}
\beta_{0}^{\ell} \\
\beta_{1}^{\ell} \\
\vdots \\
\beta_{k-2}^{\ell} \\
\beta_{k-1}^{\ell}
\end{array}\right) .
\end{aligned}
$$

But this says that

$$
\left[\begin{array}{ccc}
\left|I_{\text {left }}^{(0)}\right|_{\mu} & \cdots & \left|I_{\text {left }}^{(k-1)}\right|_{\mu} \\
\vdots & & \vdots \\
\left|I_{\text {left }}^{(k-1)}\right|_{\mu} & \cdots & \left|I_{\text {left }}^{(2 k-2)}\right|_{\mu}
\end{array}\right]\left(\begin{array}{c}
\alpha_{0}^{\ell} \\
\vdots \\
\alpha_{k-1}^{\ell}
\end{array}\right)=-\left[\begin{array}{ccc}
\left|I_{\text {right }}^{(0)}\right|_{\mu} & \cdots & \left|I_{\text {right }}^{(k-1)}\right|_{\mu} \\
\vdots & & \vdots \\
\left|I_{\text {right }}^{(k-1)}\right|_{\mu} & \cdots & \left|I_{\text {right }}^{(2 k-2)}\right|_{\mu}
\end{array}\right]\left(\begin{array}{c}
\beta_{0}^{\ell} \\
\vdots \\
\beta_{k-1}^{\ell}
\end{array}\right),
$$

for $1 \leq \ell \leq k$, which we write as

$$
\boldsymbol{L}_{I}^{k} \boldsymbol{\alpha}_{k}^{\ell}=-\boldsymbol{R}_{I}^{k} \boldsymbol{\beta}_{k}^{\ell},
$$


with

$$
\begin{aligned}
& \boldsymbol{L}_{I}^{k} \equiv\left[\begin{array}{ccccc}
\left|I_{\text {left }}^{(0)}\right|_{\mu} & \left|I_{\text {left }}^{(1)}\right|_{\mu} & \cdots & \left|I_{\text {left }}^{(k-2)}\right|_{\mu} & \left|I_{\text {left }}^{(k-1)}\right|_{\mu} \\
\left|I_{\text {left }}^{(1)}\right|_{\mu} & \left|I_{\text {left }}^{(2)}\right|_{\mu} & \cdots & \left|I_{\text {left }}^{(k-1)}\right|_{\mu} & \left|I_{\text {left }}^{(k)}\right|_{\mu} \\
\vdots & \vdots & & & \vdots \\
\left|I_{\text {left }}^{(k-2)}\right|_{\mu} & \left|I_{\text {left }}^{(k-1)}\right|_{\mu} & \cdots & \left|I_{\text {left }}^{(2 k-4)}\right|_{\mu} & \left|I_{\text {left }}^{(2 k-3)}\right|_{\mu} \\
\left|I_{\text {left }}^{(k-1)}\right|_{\mu} & \left|I_{\text {left }}^{(k)}\right|_{\mu} & \cdots & \left|I_{\text {left }}^{(2 k-3)}\right|_{\mu} & \left|I_{\text {left }}^{(2 k-2)}\right|_{\mu}
\end{array}\right], \\
& \boldsymbol{R}_{I}^{k} \equiv\left[\begin{array}{ccccc}
\left|I_{\text {right }}^{(0)}\right|_{\mu} & \left|I_{\text {right }}^{(1)}\right|_{\mu} & \cdots & \left|I_{\text {right }}^{(k-2)}\right|_{\mu} & \left|I_{\text {right }}^{(k-1)}\right|_{\mu} \\
\left|I_{\text {right }}^{(1)}\right|_{\mu} & \left|I_{\text {right }}^{(2)}\right|_{\mu} & \cdots & \left|I_{\text {right }}^{(k-1)}\right|_{\mu} & \left|I_{\text {right }}^{(k)}\right|_{\mu} \\
\vdots & \vdots & & & \vdots \\
\left|I_{\text {right }}^{(k-2)}\right|_{\mu} & \left|I_{\text {right }}^{(k-1)}\right|_{\mu} & \cdots & \left|I_{\text {right }}^{(2 k-4)}\right|_{\mu} & \left|I_{\text {right }}^{(2 k-3)}\right|_{\mu} \\
\left|I_{\text {right }}^{(k-1)}\right|_{\mu} & \left|I_{\text {right }}^{(k)}\right|_{\mu} & \cdots & \left|I_{\text {right }}^{(2 k-3)}\right|_{\mu} & \left|I_{\text {right }}^{(2 k-2)}\right|_{\mu}
\end{array}\right], \\
& \boldsymbol{\alpha}_{k}^{\ell} \equiv\left(\begin{array}{c}
\alpha_{0}^{\ell} \\
\vdots \\
\alpha_{k-1}^{\ell}
\end{array}\right), \quad \boldsymbol{\beta}_{k}^{\ell}=\left(\begin{array}{c}
\beta_{0}^{\ell} \\
\vdots \\
\beta_{k-1}^{\ell}
\end{array}\right)
\end{aligned}
$$

and by our determinant assumption we can then solve for $\boldsymbol{\alpha}_{k}^{\ell}$ in terms of $\boldsymbol{\beta}_{k}^{\ell}$;

$$
\boldsymbol{\alpha}_{k}^{\ell}=-\left[L_{I}^{k}\right]^{-1} R_{I}^{k} \boldsymbol{\beta}_{k}^{\ell}, \quad 1 \leq \ell \leq k .
$$

Thus using the Gram-Schmidt orthogonalization algorithm, there is an orthonormal basis $\left\{a_{I}^{\mu, \ell}(x)\right\}_{\ell=1}^{k}$ of $L_{Q ; k}^{2}(\mu)$ consisting of Alpert functions if and only if at least one of the matrices $\boldsymbol{L}_{I}^{k}$ and $\boldsymbol{R}_{I}^{k}$ is nonsingular (equivalently positive definite, since these symmetric matrices are always nonnegative semidefinite). For example, if we assume that $L_{I}^{k}$ is invertible, then just as in the case $k=2$ discussed above, a function $a_{I}^{\mu, \ell^{\prime}}(x)$ is orthogonal to $a_{I}^{\mu, \ell}(x)$ in $L^{2}(\mu)$, i.e.

$$
\begin{aligned}
0= & \int a_{I}^{\mu, \ell}(x) a_{I}^{\mu, \ell^{\prime}}(x) d \mu(x) \\
= & \int\left\{\left(\alpha_{k-1}^{\ell} x^{k-1}+\alpha_{k-2}^{\ell} x^{k-2}+\ldots+\alpha_{1}^{\ell} x+\alpha_{0}^{\ell}\right)\left(\alpha_{k-1}^{\ell^{\prime}} x^{k-1}+\alpha_{k-2}^{\ell^{\prime}} x^{k-2}+\ldots+\alpha_{1}^{\ell^{\prime}} x+\alpha_{0}^{\ell^{\prime}}\right) \mathbf{1}_{I_{\text {left }}}(x)\right. \\
& \left.+\left(\beta_{k-1}^{\ell} x^{k-1}+\beta_{k-2}^{\ell} x^{k-2}+\ldots+\beta_{1}^{\ell} x+\beta_{0}^{\ell}\right)\left(\beta_{k-1}^{\ell^{\prime}} x^{k-1}+\beta_{k-2}^{\ell^{\prime}} x^{k-2}+\ldots+\beta_{1}^{\ell^{\prime}} x+\beta_{0}^{\ell^{\prime}}\right) \mathbf{1}_{I_{\mathrm{right}}}(x)\right\} d \mu(x) \\
= & \left(\boldsymbol{\alpha}_{k}^{\ell}\right)^{\operatorname{tr}} \boldsymbol{L}_{I} \boldsymbol{\alpha}_{k}^{\ell^{\prime}}+\left(\boldsymbol{\beta}_{k}^{\ell}\right)^{\operatorname{tr}} \boldsymbol{R}_{I} \boldsymbol{\beta}_{k}^{\ell^{\prime}} \\
= & \left(\boldsymbol{\beta}_{k}^{\ell}\right)^{\operatorname{tr}}\left[\boldsymbol{L}_{I}^{-1} \boldsymbol{R}_{I}\right]^{\operatorname{tr}} \boldsymbol{L}_{I}\left[\boldsymbol{L}_{I}^{-1} \boldsymbol{R}_{I}\right] \boldsymbol{\beta}_{k}^{\ell^{\prime}}+\left(\boldsymbol{\beta}_{k}^{\ell}\right)^{\operatorname{tr}} \boldsymbol{R}_{I} \boldsymbol{\beta}_{k}^{\ell^{\prime}}
\end{aligned}
$$

equivalently

$$
0=\left(\boldsymbol{\beta}_{k}^{\ell}\right)^{\operatorname{tr}}\left\{\boldsymbol{R}_{I} \boldsymbol{L}_{I}^{-1} \boldsymbol{L}_{I} \boldsymbol{L}_{I}^{-1} \boldsymbol{R}_{I}+\boldsymbol{R}_{I}\right\} \boldsymbol{\beta}_{k}^{\ell^{\prime}}=\left(\boldsymbol{\beta}_{k}^{\ell}\right)^{\operatorname{tr}} \boldsymbol{R}_{I} \boldsymbol{L}_{I}^{-1}\left\{\boldsymbol{R}_{I}+\boldsymbol{L}_{I}\right\} \boldsymbol{\beta}_{k}^{\ell^{\prime}}=\left(\boldsymbol{\beta}_{k}^{\ell}\right)^{\operatorname{tr}} \boldsymbol{X}_{I} \boldsymbol{\beta}_{k}^{\ell^{\prime}},
$$

where $\boldsymbol{X}_{I} \equiv \boldsymbol{L}_{I} \boldsymbol{R}_{I} \boldsymbol{L}_{I}^{-1}\left\{\boldsymbol{R}_{I}+\boldsymbol{L}_{I}\right\}$ is invertible since $\boldsymbol{R}_{I}+\boldsymbol{L}_{I}=\boldsymbol{M}_{I, k} \succ 0$ by (3.9). Thus the orthogonality can be achieved simply by choosing $\boldsymbol{\beta}_{k}^{\ell^{\prime}}$ perpendicular to the vector $\left(\boldsymbol{\beta}_{k}^{\ell}\right)^{\operatorname{tr}} \boldsymbol{X}_{I}$.

Finally we note that the system of equations that we have solved to obtain an orthonormal basis of Alpert functions is underdetermined since we have not yet solved for the additional moment conditions,

$$
\int a_{I}^{\mu, \ell}(x) x^{i} d \mu(x)=0, \quad \text { for all } 2 \leq \ell \leq k \text { and } k \leq i \leq k+\ell-2,
$$


and we now explicitly compute the additional equations under which these additional moment conditions can also be achieved for the orthonormal basis of Alpert functions. As above have

$$
\begin{aligned}
0= & \alpha_{k-1}^{\ell}\left|I_{\text {left }}^{(i+k-1)}\right|_{\mu}+\alpha_{k-2}^{\ell}\left|I_{\text {left }}^{(i+k-2)}\right|_{\mu}+\ldots+\alpha_{0}^{\ell}\left|I_{\text {left }}^{(i)}\right|_{\mu} \\
& +\beta_{k-1}^{\ell}\left|I_{\text {right }}^{(i+k-1)}\right|_{\mu}+\beta_{k-2}^{\ell}\left|I_{\text {right }}^{(i+k-2)}\right|_{\mu}+\ldots+\beta_{0}^{\ell}\left|I_{\text {right }}^{(i)}\right|_{\mu},
\end{aligned}
$$

but now for all $2 \leq \ell \leq k$ and $k \leq i \leq k+\ell-2$, which leads to

$$
\left[\begin{array}{lll}
\left|I_{\text {left }}^{(i)}\right|_{\mu} & \cdots & \left|I_{\text {left }}^{(i+k-1)}\right|_{\mu}
\end{array}\right]\left(\begin{array}{c}
\alpha_{0}^{\ell} \\
\vdots \\
\alpha_{k-1}^{\ell}
\end{array}\right)+\left[\left|I_{\text {right }}^{(i)}\right|_{\mu} \quad \cdots \quad\left|I_{\text {right }}^{(i+k-1)}\right|_{\mu}\right]\left(\begin{array}{c}
\beta_{0}^{\ell} \\
\vdots \\
\beta_{k-1}^{\ell}
\end{array}\right)=0,
$$

for all $2 \leq \ell \leq k$ and $k \leq i \leq k+\ell-2$,

and can be written as

$$
\mathbf{v}_{\text {left }}^{(i+k-1, i)} \cdot \boldsymbol{\alpha}_{k}^{\ell}+\mathbf{v}_{\text {right }}^{(i+k-1, i)} \cdot \boldsymbol{\beta}_{k}^{\ell}=0, \quad 2 \leq \ell \leq k \text { and } k \leq i \leq k+\ell-2,
$$

where

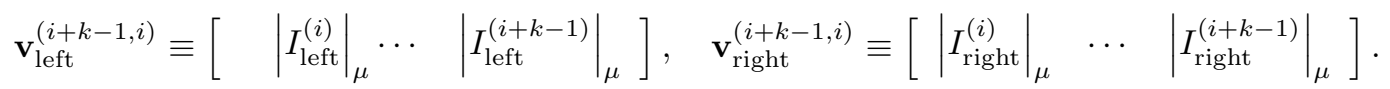

We now consider the $\left(\begin{array}{l}k \\ 2\end{array}\right)=\frac{k(k-1)}{2}$ orthogonality conditions among the $k$ Alpert functions $\left\{a_{I}^{\mu, \ell}\right\}_{\ell=1}^{k}$ and the $\left(\begin{array}{c}k \\ 2\end{array}\right)$ additional moment conditions arising from the index choices $(\ell, i)$ in (3.5) , which for convenience in visualizing we arrange in triangular form as

$$
\left\{\begin{array}{ccccccc}
(k, k) & (k, k+1) & (k, k+2) & \cdots & (k, 2 k-4) & (k, 2 k-3) & (k, 2 k-2) \\
(k-1, k) & (k-1, k+1) & (k-1, k+2) & \cdots & (k-1,2 k-4) & (k-1,2 k-3) & \\
(k-2, k) & (k-2, k+1) & (k-2, k+2) & \cdots & (k-2,2 k-4) & & \vdots \\
\vdots & \vdots & \vdots & \vdots & \vdots & \vdots & \\
(4, k) & (4, k+1) & (4, k+2) & \cdots & & & \\
(3, k) & (3, k+1) & & \cdots & & &
\end{array}\right\} .
$$

Using $\boldsymbol{\alpha}_{k}^{\ell}=-\left[\boldsymbol{L}_{I}^{k}\right]^{-1} \boldsymbol{R}_{I}^{k} \boldsymbol{\beta}_{k}^{\ell}$, the corresponding additional moment conditions are given by

$$
\begin{gathered}
0=\mathbf{v}_{\text {left }}^{(i+k-1, i)} \cdot \boldsymbol{\alpha}_{k}^{\ell}+\mathbf{v}_{\text {right }}^{(i+k-1, i)} \cdot \boldsymbol{\beta}_{k}^{\ell}=\left\{-\mathbf{v}_{\text {left }}^{(i+k-1, i)} \cdot \mathbf{M}_{I}^{k}+\mathbf{v}_{\text {right }}^{(i+k-1, i)}\right\} \cdot \boldsymbol{\beta}_{k}^{\ell}=\mathbf{w}_{k}^{i} \cdot \boldsymbol{\beta}_{k}^{\ell} \\
\text { for } 2 \leq \ell \leq k \text { and } k \leq i \leq k+\ell-2,
\end{gathered}
$$

where $\mathbf{M}_{I}^{k} \equiv-\left[\boldsymbol{L}_{I}^{k}\right]^{-1} \boldsymbol{R}_{I}^{k} \boldsymbol{\beta}_{k}^{\ell}$, and

$$
\mathbf{v}_{\text {left }}^{(i+k-1, i)} \equiv\left[\begin{array}{lll}
\left|I_{\text {left }}^{(i)}\right|_{\mu} & \cdots & \left|I_{\text {left }}^{(i+k-1)}\right|_{\mu}
\end{array}\right], \quad \mathbf{v}_{\text {right }}^{(i+k-1, i)} \equiv\left[\left.\left|\begin{array}{ll}
\left|I_{\text {right }}^{(i)}\right|_{\mu} & \cdots
\end{array}\right| I_{\text {right }}^{(i+k-1)}\right|_{\mu}\right]
$$

and

$$
\mathbf{w}_{k}^{i} \equiv-\mathbf{v}_{\text {left }}^{(i+k-1, i)} \cdot \mathbf{M}_{I}^{k}+\mathbf{v}_{\text {right }}^{(i+k-1, i)}
$$


Thus the additional moment conditions are $0=\mathbf{w}_{k}^{i} \cdot \boldsymbol{\beta}_{k}^{\ell}$ for all $2 \leq \ell \leq k$ and $k \leq i \leq k+\ell-2$, and written in the form of triangular matrix (3.6) are

$$
\begin{aligned}
0= & \mathbf{w}_{k}^{k} \cdot \boldsymbol{\beta}_{k}^{k}=\mathbf{w}_{k}^{k+1} \cdot \boldsymbol{\beta}_{k}^{k}=\ldots=\mathbf{w}_{k}^{2 k-2} \cdot \boldsymbol{\beta}_{k}^{k}, \\
0= & \mathbf{w}_{k}^{k} \cdot \boldsymbol{\beta}_{k}^{k-1}=\mathbf{w}_{k}^{k+1} \cdot \boldsymbol{\beta}_{k}^{k-1}=\ldots=\mathbf{w}_{k}^{2 k-3} \cdot \boldsymbol{\beta}_{k}^{k-1}, \\
0= & \mathbf{w}_{k}^{k} \cdot \boldsymbol{\beta}_{k}^{k-2}=\mathbf{w}_{k}^{k+1} \cdot \boldsymbol{\beta}_{k}^{k-2}=\ldots=\mathbf{w}_{k}^{2 k-4} \cdot \boldsymbol{\beta}_{k}^{k-2}, \\
& \vdots \\
0= & \mathbf{w}_{k}^{k} \cdot \boldsymbol{\beta}_{k}^{4}=\mathbf{w}_{k}^{k+1} \cdot \boldsymbol{\beta}_{k}^{4}=\mathbf{w}_{k}^{k+2} \cdot \boldsymbol{\beta}_{k}^{4}, \\
0= & \mathbf{w}_{k}^{k} \cdot \boldsymbol{\beta}_{k}^{3}=\mathbf{w}_{k}^{k+1} \cdot \boldsymbol{\beta}_{k}^{3}, \\
0= & \mathbf{w}_{k}^{k} \cdot \boldsymbol{\beta}_{k}^{2} .
\end{aligned}
$$

Now recall that mutual orthogonality of the $k$ Alpert functions $\left\{a_{I}^{\mu, \ell}\right\}_{\ell=1}^{k}$ requires choosing $\boldsymbol{\beta}_{k}^{\ell^{\prime}}$ perpendicular to the vector $\left(\boldsymbol{\beta}_{k}^{\ell}\right)^{\operatorname{tr}} \boldsymbol{X}_{I}$ for $\ell \neq \ell^{\prime}$. In addition we must have from (3.6) that

$$
\begin{aligned}
& \mathbf{w}_{k}^{k}, \mathbf{w}_{k}^{k+1}, \ldots, \mathbf{w}_{k}^{2 k-2} \in\left(\boldsymbol{\beta}_{k}^{k}\right)^{\perp}, \\
& \mathbf{w}_{k}^{k}, \mathbf{w}_{k}^{k+1}, \ldots, \mathbf{w}_{k}^{2 k-3} \in\left(\boldsymbol{\beta}_{k}^{k-1}\right)^{\perp}, \\
& \mathbf{w}_{k}^{k}, \mathbf{w}_{k}^{k+1}, \ldots, \mathbf{w}_{k}^{2 k-4} \in\left(\boldsymbol{\beta}_{k}^{k-2}\right)^{\perp}, \\
& \vdots \\
& \mathbf{w}_{k}^{k}, \mathbf{w}_{k}^{k+1}, \mathbf{w}_{k}^{k+2} \in\left(\boldsymbol{\beta}_{k}^{4}\right)^{\perp}, \\
& \mathbf{w}_{k}^{k}, \mathbf{w}_{k}^{k+1} \in\left(\boldsymbol{\beta}_{k}^{3}\right)^{\perp}, \\
& \mathbf{w}_{k}^{k} \in\left(\boldsymbol{\beta}_{k}^{2}\right)^{\perp},
\end{aligned}
$$

where $\boldsymbol{\beta}^{\perp} \equiv\left\{\mathbf{u} \in \mathbb{R}^{k}: \mathbf{u} \cdot \boldsymbol{\beta}=0\right\}$. Now each of the matrices $\boldsymbol{R}_{I} \boldsymbol{L}_{I}^{-1} \mathbf{R}_{I}$ and $\boldsymbol{R}_{I}$ is symmetric and positive definite, and so then is their sum $\boldsymbol{X}_{I}=\boldsymbol{R}_{I} \boldsymbol{L}_{I}^{-1} \boldsymbol{R}_{I}+\boldsymbol{R}_{I}$. Thus we can consider the inner product defined by

$$
\langle\mathbf{v}, \mathbf{w}\rangle_{I} \equiv \mathbf{v}^{\operatorname{tr}} \boldsymbol{X}_{I} \mathbf{w}
$$

This gives us a $k$-dimensional inner product space which we denote by $\mathcal{X}_{I}$. Now the condition $\mathbf{w}_{k}^{i} \cdot \boldsymbol{\beta}_{k}^{\ell}=0$ can be written as

$$
\left\langle\mathbf{u}_{k}^{i}, \boldsymbol{\beta}_{k}^{\ell}\right\rangle_{I}=\left(\mathbf{u}_{k}^{i}\right)^{\operatorname{tr}} \boldsymbol{X}_{I} \boldsymbol{\beta}_{k}^{\ell}=\mathbf{w}_{k}^{i} \cdot \boldsymbol{\beta}_{k}^{\ell}=0
$$

if we define $\mathbf{u}_{k}^{i}=\boldsymbol{X}_{I}^{-1}\left(\mathbf{w}_{k}^{i}\right)^{\mathrm{tr}}$. Then our conditions become

$$
\begin{aligned}
\left\langle\boldsymbol{\beta}_{k}^{\ell}, \boldsymbol{\beta}_{k}^{\ell^{\prime}}\right\rangle_{I} & =0 \text { for } 1 \leq \ell<\ell^{\prime} \leq k, \\
\left\langle\mathbf{u}_{k}^{i}, \boldsymbol{\beta}_{k}^{\ell}\right\rangle_{I} & =0 \text { for } 2 \leq \ell \leq k \text { and } k \leq i \leq k+\ell-2,
\end{aligned}
$$


and the second line above can be written out in triangular form as

$$
\begin{aligned}
& \mathbf{u}_{k}^{k}, \mathbf{u}_{k}^{k+1}, \ldots, \mathbf{u}_{k}^{2 k-2} \in\left(\boldsymbol{\beta}_{k}^{k}\right)^{\perp_{I}}, \\
& \mathbf{u}_{k}^{k}, \mathbf{u}_{k}^{k+1}, \ldots, \mathbf{u}_{k}^{2 k-3} \in\left(\boldsymbol{\beta}_{k}^{k-1}\right)^{\perp_{I}}, \\
& \mathbf{u}_{k}^{k}, \mathbf{u}_{k}^{k+1}, \ldots, \mathbf{u}_{k}^{2 k-4} \in\left(\boldsymbol{\beta}_{k}^{k-2}\right)^{\perp_{I}}, \\
& \vdots \\
& \mathbf{u}_{k}^{k}, \mathbf{u}_{k}^{k+1}, \mathbf{u}_{k}^{k+2} \in\left(\boldsymbol{\beta}_{k}^{4}\right)^{\perp_{I}}, \\
& \mathbf{u}_{k}^{k}, \mathbf{u}_{k}^{k+1} \in\left(\boldsymbol{\beta}_{k}^{3}\right)^{\perp_{I}}, \\
& \mathbf{u}_{k}^{k} \in\left(\boldsymbol{\beta}_{k}^{2}\right)^{\perp_{I}},
\end{aligned}
$$

where $\boldsymbol{\beta}^{\perp_{I}} \equiv\left\{\mathbf{u} \in \mathcal{X}_{I}:\langle\mathbf{u}, \boldsymbol{\beta}\rangle_{I}=0\right\}$.

We can satisfy conditions (3.7) by first choosing a unit vector $\boldsymbol{\beta}_{k}^{k} \in \mathcal{X}_{I}$ so that the first line in (3.8) holds. Then we choose a unit vector $\boldsymbol{\beta}_{k}^{k-1} \in\left(\boldsymbol{\beta}_{k}^{k}\right)^{\perp_{I}}$ so that the second line in (3.8) holds. Then we choose a unit vector $\boldsymbol{\beta}_{k}^{k-2} \in\left(\operatorname{Span}\left\{\boldsymbol{\beta}_{k}^{k-1}, \boldsymbol{\beta}_{k}^{k}\right\}\right)^{\perp_{I}}$ so that the third line in (3.8) holds. Continuing in this way, we find unit vectors $\left\{\boldsymbol{\beta}_{k}^{\ell}\right\}_{\ell=1}^{k}$ in the inner product space $\mathcal{X}_{I}$ so that our conditions (3.8) and (3.7) hold. For the generic choice of vectors $\mathbf{v}_{\text {left }}^{(i+k-1, i)}$ and $\mathbf{v}_{\text {right }}^{(i+k-1, i)}$, the choice of $\left\{\boldsymbol{\beta}_{k}^{\ell}\right\}_{\ell=1}^{k}$ will be unique up to sign. This completes the proof of part (2) of Theorem 2 .

We have adopted the additional moment conditions introduced by Alpert in the setting of Lebesgue measure in $\mathrm{Alp}$, but one can in fact replace these conditions by an essentially arbitrary collection of the correct number of moment conditions. We leave the nondegenerate case for the reader in the following exercise, and the degenerate case is not treated here at all, except for the simple case when $k=2$ and $n=1$ solved below.

Remark 12. If $M_{I_{\mathrm{left}, k}} \succ 0, M_{I_{\mathrm{right}}, k} \succ 0$ and $\mathcal{M} \subset\{(\ell, i): 1 \leq \ell \leq k$ and $i \geq k\}$ has cardinality $\left(\begin{array}{c}k \\ 2\end{array}\right)$, then we can choose an Alpert basis $\left\{a_{I}^{\mu, \ell}\right\}_{\ell=1}^{k}$ satisfying the additional moment conditions $\mathbf{w}_{k}^{i} \cdot \boldsymbol{\beta}_{k}^{\ell}=0$ for all $(\ell, i) \in \mathcal{M}$.

3.3.3. The Alpert degenerate case. In the event that one or more of the matrices $\boldsymbol{L}_{I}^{k}$ and/or $\boldsymbol{R}_{I}^{k}$ is singular, then it is easy to see from (3.3) that the maximum number of independent Alpert functions equals the dimension of the intersection of the ranges of $\boldsymbol{L}_{I}^{k}$ and $\boldsymbol{R}_{I}^{k}$ in $\mathbb{R}^{k}$, i.e.

$$
\operatorname{dim} L_{Q ; k}^{2}(\mu)=\operatorname{dim}\left(\text { Range } \boldsymbol{L}_{I}^{k} \bigcap \text { Range } \boldsymbol{R}_{I}^{k}\right)
$$

In the special case when $k=2$ - when there are only two Alpert functions $a_{I}^{\mu, 1}, a_{I}^{\mu, 2}$ and just one additional moment condition for $a_{I}^{\mu, 2}$ - we will show in the next subsection that it is not always possible to arrange for this additional moment condition to hold. In fact we will show there that it holds if and only if

$$
\left(\begin{array}{l}
\left|I_{\text {right }}^{(2)}\right|_{\mu} \\
\left|I_{\text {right }}^{(3)}\right|_{\mu}
\end{array}\right) \in \operatorname{Range} \boldsymbol{R}_{I}^{2} .
$$

Similar results hold for larger $k$, but we will not pursue these here.

3.4. The special case $n=1$ and $k=2$. First we quickly review and set notation for the nondegenerate case when $k=2$, and later proceed to the degenerate case. Let $\mu$ be a locally finite positive Borel measure 
on $\mathbb{R}$ that satisfies the Haar nondegeneracy condition $|J|_{\mu}>0$ for all $J \in \mathcal{D}$ and in addition satisfies the Alpert nondegeneracy condition

$$
\operatorname{det}\left[\begin{array}{ll}
\left|J^{(0)}\right|_{\mu} & \left|J^{(1)}\right|_{\mu} \\
\left|J^{(1)}\right|_{\mu} & \left|J^{(2)}\right|_{\mu}
\end{array}\right]>0, \quad \text { for all } J \in \mathcal{D} .
$$

Recall that the determinant in (3.9) is nonnegative by the Cauchy-Schwarz inequality, and is positive if and only if the functions $\mathbf{1}_{J}(x)$ and $x \mathbf{1}_{J}(x)$ are linearly independent on $J$ :

$$
\left|J^{(1)}\right|_{\mu}^{2}=\left(\int_{J} x d \mu(x)\right)^{2} \leq\left(\int_{J} 1^{2} d \mu(x)\right)\left(\int_{J} x^{2} d \mu(x)\right)=\left|J^{(0)}\right|_{\mu}\left|J^{(2)}\right|_{\mu}
$$

with equality if and only if the functions $\mathbf{1}_{J}(x)$ and $x \mathbf{1}_{J}(x)$ are linearly dependent on $J$.

We require the functions

$$
\begin{aligned}
& a_{I}^{\mu, 1}(x) \equiv\left(\alpha_{1}^{1} x+\alpha_{0}^{1}\right) \mathbf{1}_{I_{\text {left }}}(x)+\left(\beta_{1}^{1} x+\beta_{0}^{1}\right) \mathbf{1}_{I_{\mathrm{right}}}(x), \\
& a_{I}^{\mu, 2}(x) \equiv\left(\alpha_{1}^{2} x+\alpha_{0}^{2}\right) \mathbf{1}_{I_{\mathrm{left}}}(x)+\left(\beta_{1}^{2} x+\beta_{0}^{2}\right) \mathbf{1}_{I_{\mathrm{right}}}(x),
\end{aligned}
$$

to satisfy the moment properties,

$$
\begin{aligned}
& \text { (A) } \int a_{I}^{\mu, 1}(x) d \mu(x)=0 \text { and } \int a_{I}^{\mu, 1}(x) x d \mu(x)=0, \\
& \text { (B) } \int a_{I}^{\mu, 2}(x) d \mu(x)=0 \text { and } \int a_{I}^{\mu, 2}(x) x d \mu(x)=0, \\
& \text { (C) } \int a_{I}^{\mu, 2}(x) x^{2} d \mu(x)=0,
\end{aligned}
$$

the orthogonality property,

$$
\text { (D) } \int a_{I}^{\mu, 1}(x) a_{I}^{\mu, 2}(x) d \mu(x)=0
$$

and the normalization properties,

$$
\begin{aligned}
& \left(\int\left|a_{I}^{\mu, 1}(x)\right|^{2} d \mu(x)\right)^{\frac{1}{2}}=1, \\
& \left(\int\left|a_{I}^{\mu, 2}(x)\right|^{2} d \mu(x)\right)^{\frac{1}{2}}=1 .
\end{aligned}
$$

Theorem 1 above gives the following conclusion.

Theorem 13. The collection

is an orthonormal basis for $L^{2}(\mu)$.

$$
\mathcal{U}^{\mathrm{Alpert}, \mu} \equiv\left\{a_{I}^{\mu, 1}, a_{I}^{\mu, 2}\right\}_{I \in \mathcal{D}}
$$

If we wish to include the additional moment condition (C), i.e. $\int a_{I}^{\mu, 2}(x) x^{2} d \mu(x)=0$, then we must in addition solve

$$
\begin{aligned}
0 & =\int\left\{\left(\alpha_{1}^{2} x+\alpha_{0}^{2}\right) \mathbf{1}_{I_{\text {left }}}(x)+\left(\beta_{1}^{2} x+\beta_{0}^{2}\right) \mathbf{1}_{I_{\text {right }}}(x)\right\} x^{2} d \mu(x) \\
& =\alpha_{0}^{2}\left|I_{\text {left }}^{(2)}\right|_{\mu}+\alpha_{1}^{2}\left|I_{\text {left }}^{(3)}\right|_{\mu}+\beta_{0}^{2}\left|I_{\text {right }}^{(2)}\right|_{\mu}+\beta_{1}^{2}\left|I_{\text {right }}^{(3)}\right|_{\mu} \\
& =\left(\left|I_{\text {left }}^{(2)}\right|_{\mu}\left|I_{\text {left }}^{(3)}\right|_{\mu}\right)\left(\begin{array}{c}
\alpha_{0}^{2} \\
\alpha_{1}^{2}
\end{array}\right)+\left(\left|I_{\text {right }}^{(2)}\right|_{\mu}\left|I_{\text {right }}^{(3)}\right|_{\mu}\right)\left(\begin{array}{c}
\beta_{0}^{2} \\
\beta_{1}^{2}
\end{array}\right) \\
& =\left\{-\left(\left|I_{\text {left }}^{(2)}\right|_{\mu}\left|I_{\text {left }}^{(3)}\right|_{\mu}\right) L_{I}^{-1} R_{I}+\left(\mid \begin{array}{ll}
\left.I_{\text {right }}^{(2)}\right|_{\mu}\left|I_{\text {right }}^{(3)}\right|_{\mu}
\end{array}\right)\right\}\left(\begin{array}{c}
\beta_{0}^{2} \\
\beta_{1}^{2}
\end{array}\right)
\end{aligned}
$$

which means we must choose $\left(\begin{array}{c}\beta_{0}^{2} \\ \beta_{1}^{2}\end{array}\right)$ perpendicular to the vector

$$
-\left(\left|I_{\text {left }}^{(2)}\right|_{\mu}\left|I_{\text {left }}^{(3)}\right|_{\mu}\right) \boldsymbol{L}_{I}^{-1} \boldsymbol{R}_{I}+\left(\left|I_{\text {right }}^{(2)}\right|_{\mu}\left|I_{\text {right }}^{(3)}\right|_{\mu}\right) .
$$


Thus it must be the case that the two vectors

$$
\left(\begin{array}{cc}
\beta_{0}^{1} & \beta_{1}^{1}
\end{array}\right) \boldsymbol{R}_{I} \boldsymbol{L}_{I}^{-1}\left\{\boldsymbol{R}_{I}+\boldsymbol{L}_{I}\right\}
$$

and

$$
-\left(\left|I_{\text {left }}^{(2)}\right|_{\mu}\left|I_{\text {left }}^{(3)}\right|_{\mu}\right) L_{I}^{-1} R_{I}+\left(\left|I_{\text {right }}^{(2)}\right|_{\mu}\left|I_{\text {right }}^{(3)}\right|_{\mu}\right)
$$

are parallel. But since $\boldsymbol{R}_{I} \boldsymbol{L}_{I}^{-1}\left\{\boldsymbol{R}_{I}+\boldsymbol{L}_{I}\right\}$ is invertible, this can clearly be achieved by choosing $\left(\begin{array}{ll}\beta_{0}^{1} & \beta_{1}^{1}\end{array}\right)$ appropriately, thereby using up our last degree of freedom in the case $k=2$.

Finally, we examine what happens when one or more of the nondegeneracy conditions (2.1) and (3.9) fails. Note that for a given interval $J$, we have that (2.1) holds and (3.9) fails if and only if $\mu 1_{J}$ is a point mass. Indeed, $\mathbf{1}_{J}(x)$ and $x \mathbf{1}_{J}(x)$ are dependent if and only if $\mu 1_{J}$ is a point mass, which we locate at $x_{J} \in J$. In this case we set

$$
\mu 1_{J}=|J|_{\mu} \delta_{x_{J}}
$$

We also recall

$$
\boldsymbol{M}_{J}=\boldsymbol{M}_{J, 2} \equiv\left[\begin{array}{ll}
\left|J_{\text {left }}^{(0)}\right|_{\mu} & \left|J_{\text {left }}^{(1)}\right|_{\mu} \\
\left|J_{\text {left }}^{(1)}\right|_{\mu} & \left|J_{\text {left }}^{(2)}\right|_{\mu}
\end{array}\right] \text { for } J \in \mathcal{D} .
$$

Lemma 14. Let $I=I_{\text {left }} \dot{\cup} I_{\text {right }}$ be the decomposition of $I$ into its two children.

(1) If (2.1) holds for both $I_{\text {left }}$ and $I_{\text {right }}$, and (3.9) holds for $I_{\text {left }}$ but fails for $I_{\text {right }}$, then $a_{I}^{\mu, 1}=a_{I}^{\mu, 2} \neq 0$ in $L^{2}(\mu)$. This conclusion persists in the opposite situation where (2.1) holds for both $I_{\text {left }}$ and $I_{\text {right }}$, but (3.9) fails for $I_{\text {left }}$ and holds for $I_{\text {right }}$.

(2) In all other degenerate cases, where at least one of the matrices $\left[\begin{array}{cc}\left|\left(I_{\text {left }}\right)^{(0)}\right|_{\mu} & \left|\left(I_{\text {left }}\right)^{(1)}\right|_{\mu} \\ \left|\left(I_{\text {left }}\right)^{(1)}\right|_{\mu} & \left|\left(I_{\text {left }}\right)^{(2)}\right|_{\mu}\end{array}\right]$, $\left[\begin{array}{ll}\left|\left(I_{\text {right }}\right)^{(0)}\right|_{\mu} & \left|\left(I_{\text {right }}\right)^{(1)}\right|_{\mu} \\ \left|\left(I_{\text {right }}\right)^{(1)}\right|_{\mu} & \left|\left(I_{\text {right }}\right)^{(2)}\right|_{\mu}\end{array}\right]$ fails to be positive definite, we have $a_{I}^{\mu, 1}=a_{I}^{\mu, 2}=0$ in $L^{2}(\mu)$.

Proof. Fix $I \in \mathcal{D}$.

Assertion (1): We have

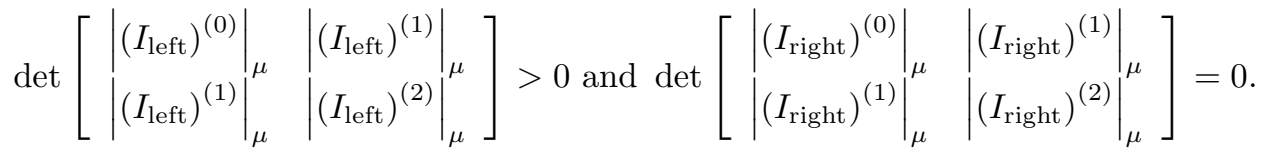

Thus $\mathbf{1}_{I_{j, \mathrm{right}}^{m}} \mu$ is a point mass located at $x_{I_{j, \mathrm{right}}^{m}}$. Now (3.4) shows that $\left(\begin{array}{c}\alpha_{0}^{\ell} \\ \alpha_{1}^{\ell}\end{array}\right)$ is uniquely determined by $\left(\begin{array}{c}\beta_{0}^{\ell} \\ \beta_{1}^{\ell}\end{array}\right)$ for $1 \leq \ell \leq 2$, and we know that the range of $\left[\begin{array}{ll}\left|\left(I_{j, \text { right }}^{m}\right)^{(2)}\right|_{\mu} & \left|\left(I_{j \text {,right }}^{m}\right)^{(1)}\right|_{\mu} \\ \left|\left(I_{j, \text { right }}^{m}\right)^{(1)}\right|_{\mu} & \left|\left(I_{j \text {,right }}^{m}\right)^{(0)}\right|_{\mu}\end{array}\right]$ is just onedimensional, since $\mathbf{1}_{I_{j, \mathrm{right}}^{m}}^{m} \mu$ is a point mass located at $x_{I_{j, \mathrm{right}}^{m}}$. It follows that both $a_{I}^{\mu, 1}$ and $a_{I}^{\mu, 2}$ are constant on the interval $I_{j, \text { right }}^{m}$ in the space $L^{2}(\mu)$, and since the null space of $\left[\begin{array}{lll}\left|\left(I_{j, \text { right }}^{m}\right)^{(0)}\right|_{\mu} & \left|\left(I_{j, \text { right }}^{m}\right)^{(1)}\right|_{\mu} \\ \left|\left(I_{j, \text { right }}^{m}\right)^{(1)}\right|_{\mu} & \left|\left(I_{j, \text { right }}^{m}\right)^{(2)}\right|_{\mu}\end{array}\right]_{\text {is }}$ one-dimensional, namely $\operatorname{Span}\left(\begin{array}{c}-x_{I_{j, \mathrm{right}}^{m}} \\ 1\end{array}\right)$, we see that the functions $a_{I}^{\mu, 1}$ and $a_{I}^{\mu, 2}$ are linearly dependent, i.e. there is only one Alpert function in this case, i.e. $a_{I_{j}^{m}}^{\mu, 1}=a_{I_{j}^{m}}^{\mu, 2}$.

Assertion (2): From above we have $\operatorname{dim} L_{Q ; 2}^{2}(\mu)=\operatorname{dim}\left(\operatorname{Range} \boldsymbol{L}_{I}^{2} \cap \operatorname{Range} \boldsymbol{R}_{I}^{2}\right)$. Thus if one of the ranges is $\{0\}$ we are done. So we are left with the case where (2.1) holds for both $I_{j, \text { left }}^{m}$ and $I_{j, \text { right }}^{m}$, and (3.9) fails 
for both $I_{j, \text { left }}^{m}$ and $I_{j \text {,right }}^{m}$. We then have

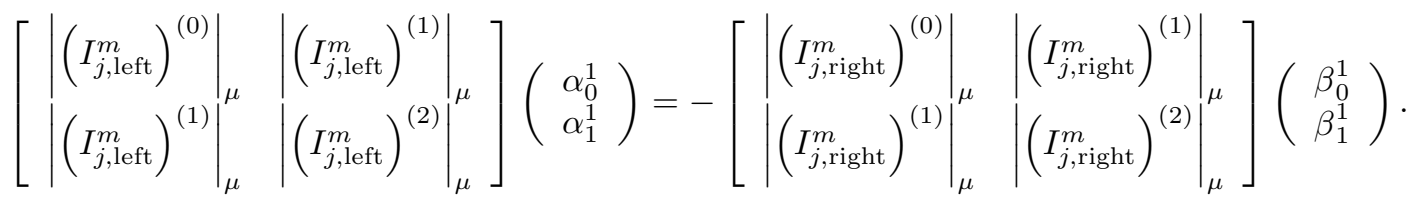

Now the point mass $\mathbf{1}_{I_{\text {right }}} \mu$ is located at $x_{I_{j, \text { right }}^{m}}$, and the point mass $\mathbf{1}_{I_{\text {left }}} \mu$ is located at $x_{I_{j, \text { left }}^{m}}$, and thus

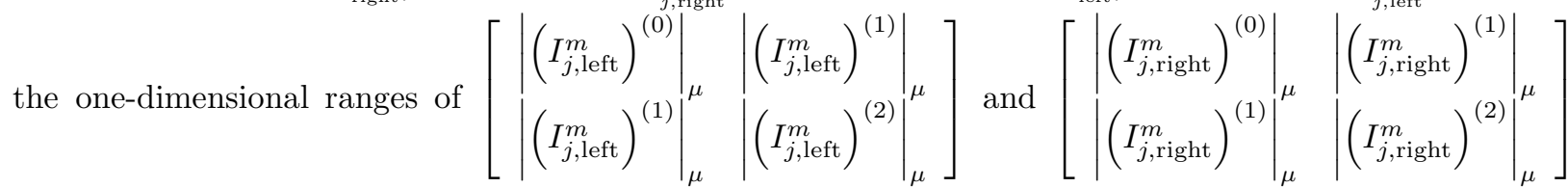
are respectively spanned by the vectors $\left(\begin{array}{c}x_{I_{j, \text { left }}^{m}} \\ 1\end{array}\right)$ and $\left(\begin{array}{c}x_{I_{j, \text { right }}^{m}} \\ 1\end{array}\right)$, which are independent since $I_{j \text {,left }}^{m} \cap$ $I_{j \text {,right }}^{m}=\emptyset$. Thus only the trivial solution $\left(\begin{array}{c}\alpha_{0}^{1} \\ \alpha_{1}^{1}\end{array}\right)=\left(\begin{array}{c}\beta_{0}^{1} \\ \beta_{1}^{1}\end{array}\right)=\left(\begin{array}{l}0 \\ 0\end{array}\right)$ exists, and it follows that both $a_{I_{j}^{m}}^{\mu, 1}$ and $a_{I_{j}^{m}}^{\mu, 2}$ vanish in $L^{2}(\mu)$.

The resulting pared collection

$$
\mathcal{U}^{\text {Alpert }, \mu} \equiv\left\{a_{I}^{\mu, 1}, a_{I}^{\mu, 2}\right\}_{I \in \mathcal{D}}
$$

where $a_{I}^{\mu, 1}$ or $a_{I}^{\mu, 2}$ or both are removed according to the lemma above, is an orthonormal basis for $L^{2}(\mu)$.

Finally, we consider the additional moment condition (C) in the case where assertion (1) of the above lemma holds, namely when (2.1) holds for both $I_{j, \text { left }}^{m}$ and $I_{j \text {,right, }}^{m}$ and (3.9) holds for $I_{j \text {,left }}^{m}$ but fails for $I_{j, \text { right }}^{m}$. In this case $a_{I_{j}^{m}}^{\mu}=b_{I_{j}^{m}}^{\mu} \neq 0$ in $L^{2}(\mu)$, and this is the only case in which there is just one Alpert function (apart from the symmetric case when (3.9) holds for $I_{j \text {,right }}^{m}$ but fails for $I_{j, \text { left }}^{m}$ ).

From the calculations above we must have that the two vectors

$$
\left(\begin{array}{ll}
\beta_{0}^{1} & \beta_{1}^{1}
\end{array}\right) \boldsymbol{R}_{I} \boldsymbol{L}_{I}^{-1}\left\{\boldsymbol{R}_{I}+\boldsymbol{L}_{I}\right\}
$$

and

$$
-\left(\left|I_{\text {left }}^{(2)}\right|_{\mu}\left|I_{\text {left }}^{(3)}\right|_{\mu}\right) \boldsymbol{L}_{I}^{-1} \boldsymbol{R}_{I}+\left(\left|I_{\text {right }}^{(2)}\right|_{\mu}\left|I_{\text {right }}^{(3)}\right|_{\mu}\right)
$$

are parallel. But since $\boldsymbol{R}_{I} \boldsymbol{L}_{I}^{-1}\left\{\boldsymbol{R}_{I}+\boldsymbol{L}_{I}\right\}$ is no longer invertible - indeed, $\boldsymbol{R}_{I} \boldsymbol{L}_{I}^{-1}\left\{\boldsymbol{R}_{I}+\boldsymbol{L}_{I}\right\}$ has rank 1 since $\boldsymbol{R}_{I}$ does - we cannot necessarily solve

$$
\left(\begin{array}{ll}
\beta_{0}^{1} & \beta_{1}^{1}
\end{array}\right)\left\{\boldsymbol{R}_{I} \boldsymbol{L}_{I}^{-1} \boldsymbol{R}_{I}+\boldsymbol{R}_{I}\right\}=\lambda\left\{\left(\left|I_{\text {right }}^{(2)}\right|_{\mu}\left|I_{\text {right }}^{(3)}\right|_{\mu}\right)-\left(\left|I_{\text {left }}^{(2)}\right|_{\mu}\left|I_{\text {left }}^{(3)}\right|_{\mu}\right) \boldsymbol{L}_{I}^{-1} \boldsymbol{R}_{I}\right\} .
$$

In fact, there is a solution if and only if the transpose of the vector

$$
\left(\left|I_{\text {right }}^{(2)}\right|_{\mu}\left|I_{\text {right }}^{(3)}\right|_{\mu}\right)-\left(\left|I_{\text {left }}^{(2)}\right|_{\mu}\left|I_{\text {left }}^{(3)}\right|_{\mu}\right) \boldsymbol{L}_{I}^{-1} \boldsymbol{R}_{I}
$$

lies in the one-dimensional range of the matrix $R_{I}$, which in turn holds if and only if

$$
\left(\begin{array}{l|l}
\mid I_{\text {right }}^{(2)} & \\
\left|I_{\text {right }}^{(3)}\right|_{\mu}
\end{array}\right) \in \text { Range } \boldsymbol{R}_{I}
$$

Remark 15. The authors thank Fletcher Gates for showing them that, in the case under consideration, this latter condition is always satisfied.

\section{Applichtion: a two weight Tp Conjecture}

Using the weighted Alpert wavelet bases constructed in Theorem 1, we can formulate an associated $T p$-type theorem in dimension $n=1$ where testing over indicators is replaced by testing over indicators times polynomials of degree at most $k-1$, and the energy condition is replaced by an associated $k$-energy condition. Unfortunately, at this point in time, we cannot demonstrate that Conjecture 16 below produces new interesting weighted inequalities, despite that fact that we provide an example to show that the $k$ energy condition is strictly weaker than the usual energy condition, even in the presence of the Muckenhoupt 
condition. But see Subsection 4.4 for a demonstration that Conjecture [16] differs 'logically' from existing T1-type theorems in the literature.

Let $0 \leq \alpha<1, k \in \mathbb{N}$ and $0<\delta<1$. We define a $(k+\delta)$-smooth $\alpha$-fractional CZ kernel $K^{\alpha}(x, y)$ to be a real-valued function defined on $\mathbb{R} \times \mathbb{R}$ satisfying the following fractional size and smoothness conditions of order $1+\delta$ : For $x \neq y$,

$$
\begin{aligned}
& \left|K^{\alpha}(x, y)\right| \leq C_{C Z}|x-y|^{\alpha-1} \text { and }\left|\nabla^{j} K^{\alpha}(x, y)\right| \leq C_{C Z}|x-y|^{\alpha-1-j}, \quad 1 \leq j \leq k, \\
& \left|\nabla^{k} K^{\alpha}(x, y)-\nabla^{k} K^{\alpha}\left(x^{\prime}, y\right)\right| \leq C_{C Z}\left(\frac{\left|x-x^{\prime}\right|}{|x-y|}\right)^{\delta}|x-y|^{\alpha-1-k}, \quad \frac{\left|x-x^{\prime}\right|}{|x-y|} \leq \frac{1}{2},
\end{aligned}
$$

and the last inequality also holds for the adjoint kernel in which $x$ and $y$ are interchanged. We associate a corresponding Calderón-Zygmund operator $T^{\alpha}$ is the usual way (see e.g. [LaWi] or [SaShUr7]).

The following conjectured $T p$ theorem with an energy side condition differs from the corresponding $T 1$ theorem with an energy side condition in two ways:

(1) Because of the stronger moment conditions satisfied Alpert wavelets, the usual energy condition assumption from the $T 1$ theorem can be weakened in the $T p$ theorem.

(2) Due to the weaker telescoping identities satisfied by Alpert wavelets, the $T 1$ testing conditions must be strengthened to testing polynomials times indicators.

Conjecture 16. Let $0 \leq \alpha<1$ and $0<\delta<1$. Let $T^{\alpha}$ be a $(k+\delta)$-smooth $\alpha$-fractional Calderón-Zygmund operator on the real line. Suppose $\sigma$ and $\omega$ are locally finite positive Borel measures on $\mathbb{R}$ that satisfy the $k$-energy condition

$$
\left(\mathcal{E}_{2, k}^{\alpha}\right)^{2} \equiv \sup _{I=\dot{\cup}_{r=1}^{\infty} I_{r}} \frac{1}{|I|_{\sigma}} \sum_{r=1}^{\infty}\left(\frac{\mathrm{P}_{k}^{\alpha}\left(I_{r}, \mathbf{1}_{I} \sigma\right)}{\left|I_{r}\right|^{k}}\right)^{2}\left\|\left(x-m_{I_{r}}^{k}\right)^{k}\right\|_{L^{2}\left(\mathbf{1}_{I_{r}} \omega\right)}^{2},
$$

as well as the dual $k$-energy condition obtained by interchanging the measures $\sigma$ and $\omega$. Then the operator $T_{\sigma}^{\alpha} f \equiv T^{\alpha}(f \sigma)$ is bounded from $L^{2}(\sigma)$ to $L^{2}(\omega)$ (in the sense that tangent line truncations are uniformly bounded by a constant $\left.\mathfrak{N}_{T^{\alpha}}(\sigma, \omega)\right)$ if

(1) there is a positive constant $\mathfrak{T}_{T^{\alpha}}^{k}(\sigma, \omega)$ such that

$$
\int_{Q}\left|T_{\sigma}^{\alpha} \mathbf{1}_{Q} p\right|^{2} d \omega \leq\left(\mathfrak{T}_{T^{\alpha}}^{k}(\sigma, \omega)\right)^{2} \int_{Q}|p|^{2} d \sigma,
$$

for all intervals $Q$ and polynomials $p(x)=c_{0}+c_{1} x+\ldots+c_{k-1} x^{k-1}$ of degree at most $k-1$, as well as the dual testing conditions obtained by interchanging the measures $\sigma$ and $\omega$ and replacing $T_{\sigma}^{\alpha}$ with its dual $T_{\omega}^{\alpha}$; and

(2) the fractional Muckenhoupt condition is finite: $\mathfrak{A}_{2}^{\alpha}(\sigma, \omega)<\infty$.

A proof of this conjecture would follow the somewhat standard lines of proof for T1-type theorems already in the literature ([NTV4], LaSaShUr3], Lac], Hyt2]), namely an inner product $\left\langle T_{\sigma}^{\alpha} f, g\right\rangle_{L^{2}(\omega)}$ is expanded in Alpert projections as

$$
\left\langle T_{\sigma}^{\alpha} f, g\right\rangle_{L^{2}(\omega)}=\sum_{\substack{I \in \mathcal{D} \\ 1 \leq \ell \leq k}} \sum_{\substack{J \in \mathcal{G} \\ 1 \leq \ell^{\prime} \leq k^{\prime}}}\left\langle T_{\sigma}^{\alpha} \triangle_{I}^{\sigma, \ell} f, \triangle_{J}^{\omega, \ell^{\prime}} g\right\rangle_{L^{2}(\omega)},
$$

and then decomposed into many separate infinite sums according to the relative sizes, locations and goodness of the intervals $I$ and $J$, which are then all controlled differently. We first highlight the two main points of departure in controlling these different sums, followed by a brief description of the sums themselves and how they are handled in the Tp situation, as well as pointing to an obstruction to the proof.

- The estimate for norms of Alpert projections $\left\|\triangle_{J}^{\omega} T^{\alpha} \mu\right\|_{L^{2}(\omega)}^{2}$, called the Monotonicity Lemma below and in LaWi], SaShUr7, is improved by the extra vanishing moments of Alpert wavelets to

$$
\left\|\triangle_{J}^{\omega} T^{\alpha} \mu\right\|_{L^{2}(\omega)}^{2} \lesssim\left(\frac{\mathrm{P}_{k}^{\alpha}(J, \mu)}{|J|^{k}}\right)^{2}\left\|\left(x-m_{J}^{k}\right)^{k}\right\|_{L^{2}\left(\mathbf{1}_{J} \omega\right)}^{2},
$$

which in turn can then be controlled by the $k$-energy condition (4.2) above, weaker than the usual energy condition with $k=1$; 
- The telescoping identities (1.1) reduce sums of consecutive Alpert projections $\triangle_{I ; k}^{\mu}$ to projections $\mathbb{E}_{Q ; k}^{\mu}$ onto spaces of polynomials of degree at most $k-1$, thus requiring the use of stronger testing conditions, taken locally over polynomials of degree at most $k-1$, in order to the bound the consecutive sums of Alpert projections that arise in the paraproduct and stopping forms in LaWi and SaShUr7.

With these two changes in mind we can now review the main steps in the standard proof strategy for the interested reader, whom we alert to the fact that we are using here the formulation of the Lacey-Wick monotonicity lemma with an error term in [LaWi] and [SaShUr7, as opposed to the stronger formulation used in LaSaShUr3 and Lac that exploited special properties of the Haar basis to hide the error term. As a consequence, the reader can follow the broad outline of the one-dimensional proof in LaSaShUr3 and [Lac, but handling the error terms as in LaWi] and/or [SaShUr7] (see also SaShUr9], SaShUr10]). We ignore the case of common point masses, and refer the reader instead to Hyt2, LaWi] and SaShUr10.

Step 1: Using the random grids of Nazarov, Treil and Volberg, a reduction is made to good functions $f$ and $g$, i.e. those whose wavelet expansions involve only cubes from one grid that are good with respect to the other grid. The orthogonality of Alpert projections plays a key role here.

Step 2: Using the testing conditions, one further restricts the supports of $f$ and $g$ to a finite union of large cubes.

Step 3: Then one can implement corona constructions with Calderón-Zygmund stopping times on the averages of $f$, and with $k$-energy stopping times in place of the familiar energy stopping times.

Step 4: The sum of inner products in (4.3) is then grouped into coronas relative to these stopping times and further decomposed into global, local and error pieces.

Step 5: The error pieces are handled by NTV methods from NTV4.

Step 6: The global inner products are controlled by the $k$-Poisson operator as in LaWi and/or SaShUr7, which in turn has its norm inequality controlled by $k$-Poisson testing conditions. This latter result is proved in the same way as is done for the familiar Poisson operator.

Step 7: The local terms are handled by Lacey's bottom/up stopping time and recursion as in Lac, with error terms from the monotonicity lemma handled as in [LaWi] and/or [SaShUr7]. The difficulty lies in using the Nazarov, Treil, Volberg method connecting back to the appropriate paraproduct terms; at the moment we are unable to control those terms and so do not have a proof of Conjecture 16.

Remark 17. There is an analogous conjecture in higher dimensions following the arguments in LaWi] and/or [SaShUr7], but we will not pursue this.

4.1. The Monotonicity Lemma. For $0 \leq \alpha<1$ and $m \in \mathbb{R}_{+}$, we recall the $m$-weighted fractional Poisson integral

$$
\mathrm{P}_{m}^{\alpha}(J, \mu) \equiv \int_{\mathbb{R}} \frac{|J|^{m}}{\left(|J|+\left|y-c_{J}\right|\right)^{m+1-\alpha}} d \mu(y),
$$

where $\mathrm{P}_{1}^{\alpha}(J, \mu)=\mathrm{P}^{\alpha}(J, \mu)$ is the standard Poisson integral.

Lemma 18 (Monotonicity). Suppose that $I$ and $J$ are cubes in $\mathbb{R}$ such that $J \subset 2 J \subset I$, and that $\mu$ is a signed measure on $\mathbb{R}$ supported outside $I$. Finally suppose that $T^{\alpha}$ is a standard fractional singular integral on $\mathbb{R}$ with kernel $K^{\alpha}(x, y)=K_{y}^{\alpha}(x), 0<\alpha<1$. Then there is a positive constant $C_{\alpha}$ such that

$$
\Phi^{\alpha}(J, \mu)^{2}-C_{\alpha} \Psi^{\alpha}(J,|\mu|)^{2} \leq\left\|\triangle_{J}^{\omega} T^{\alpha} \mu\right\|_{L^{2}(\omega)}^{2} \lesssim \Phi^{\alpha}(J, \mu)^{2}+C_{\alpha} \Psi^{\alpha}(J,|\mu|)^{2},
$$

where for a measure $\nu$,

$$
\begin{aligned}
& \Phi^{\alpha}(J, \nu)^{2} \equiv\left|\frac{1}{k !} \int\left(K_{y}^{\alpha}\right)^{(k)}\left(m_{J}\right) d \mu(y)\right|^{2}\left\|\triangle_{J}^{\omega} x^{k}\right\|_{L^{2}(\omega)}^{2}, \\
& \Psi^{\alpha}(J, \nu)^{2} \equiv\left(\frac{\mathrm{P}_{k+\delta}^{\alpha}(J, \nu)}{|J|^{k}}\right)^{2}\left\|\left(x-m_{J}^{k}\right)^{k}\right\|_{L^{2}\left(\mathbf{1}_{J} \omega\right)}^{2}, \\
& \text { where } m_{J}^{k} \in J \text { satisfies }\left\|\left(x-m_{J}^{k}\right)^{k}\right\|_{L^{2}\left(\mathbf{1}_{J} \omega\right)}^{2}=\inf _{c \in J}\left\|(x-c)^{k}\right\|_{L^{2}\left(\mathbf{1}_{J} \omega\right)}^{2} .
\end{aligned}
$$


and where if $\nu$ is a positive measure, then there are positive constants $c, C$ such that

$$
c \Phi^{\alpha}(J, \nu)^{2} \leq\left(\frac{\mathrm{P}_{k}^{\alpha}(J, \nu)}{|J|^{k}}\right)^{2}\left\|\triangle_{J}^{\omega} x^{k}\right\|_{L^{2}(\omega)}^{2} \leq C \Phi^{\alpha}(J, \nu)^{2}
$$

Remark 19. The right hand side of (4.4) is what determines the definition of the $k$-energy condition (4.2) used in the stopping time arguments adapted from [LaWi] and/or [SaShUr7].

Proof of Lemma 18. The proof is an easy adaptation of the proofs in LaWi and SaShUr7 restricted to dimension $n=1$, but using an order $k$ Taylor expansion instead of an order 1 expansion on the kernel $\left(K_{y}^{\alpha}\right)(x)=K^{\alpha}(x, y)$. Due to the importance of this lemma, as explained in the above remark, we give the short argument.

Let $\left\{h_{J}^{\omega, a}\right\}_{a \in \Gamma}$ be an orthonormal basis of $L_{J ; k}^{2}(\mu)$ consisting of Alpert functions as above. Now we use the $(k+\delta)$-smooth Calderón-Zygmund smoothness estimate (4.1), together with Taylor's formula

$$
\begin{aligned}
K_{y}^{\alpha}(x) & =T\left(K_{y}^{\alpha}\right)(x, c)+\frac{1}{k !}\left(K_{y}^{\alpha}\right)^{(k)}(\theta(x, c))(x-c)^{k} \\
\operatorname{Tay}\left(K_{y}^{\alpha}\right)(x, c) & \equiv K_{y}^{\alpha}(c)+\left(K_{y}^{\alpha}\right)^{\prime}(c)(x-c)+\ldots+\frac{1}{(k-1) !}\left(K_{y}^{\alpha}\right)^{(k-1)}(c)(x-c)^{k-1},
\end{aligned}
$$

and the vanishing means of the Alpert functions $h_{J}^{\omega, a}$, to obtain

$$
\begin{aligned}
\left\langle T^{\alpha} \mu, h_{J}^{\omega, a}\right\rangle_{\omega}= & \int\left\{\int K^{\alpha}(x, y) h_{J}^{\omega, a}(x) d \omega(x)\right\} d \mu(y)=\int\left\langle K_{y}^{\alpha}, h_{J}^{\omega, a}\right\rangle_{\omega} d \mu(y) \\
= & \int\left\langle K_{y}^{\alpha}(x)-\operatorname{Tay}\left(K_{y}^{\alpha}\right)\left(x, m_{J}^{k}\right), h_{J}^{\omega, a}(x)\right\rangle_{\omega} d \mu(y) \\
= & \int\left\langle\frac{1}{k !}\left(K_{y}^{\alpha}\right)^{(k)}\left(\theta\left(x, m_{J}^{k}\right)\right)\left(x-m_{J}^{k}\right)^{k}, h_{J}^{\omega, a}(x)\right\rangle_{\omega} d \mu(y) \\
= & \left.\left\langle\iint \frac{1}{k !}\left(K_{y}^{\alpha}\right)^{(k)}\left(m_{J}\right) d \mu(y)\right]\left(x-m_{J}^{k}\right)^{k}, h_{J}^{\omega, a}\right\rangle_{\omega} \\
& \left.+\left\langle\iint \frac{1}{k !}\left[\left(K_{y}^{\alpha}\right)^{(k)}\left(\theta\left(x, m_{J}^{k}\right)\right)-\left(K_{y}^{\alpha}\right)^{(k)}\left(m_{J}^{k}\right)\right] d \mu(y)\right]\left(x-m_{J}^{k}\right)^{k}, h_{J}^{\omega, a}\right\rangle_{\omega} \\
= & {\left[\frac{1}{k !} \int\left(K_{y}^{\alpha}\right)^{(k)}\left(m_{J}\right) d \mu(y)\right]\left\langle x^{k}, h_{J}^{\omega, a}\right\rangle_{\omega} } \\
& +\left\langle\left[\int \frac{1}{k !}\left[\left(K_{y}^{\alpha}\right)^{(k)}\left(\theta\left(x, m_{J}^{k}\right)\right)-\left(K_{y}^{\alpha}\right)^{(k)}\left(m_{J}^{k}\right)\right] d \mu(y)\right]\left(x-m_{J}^{k}\right)^{k}, h_{J}^{\omega, a}\right\rangle_{\omega}
\end{aligned}
$$

and hence

$$
\begin{aligned}
& \left|\left\langle T^{\alpha} \mu, h_{J}^{\omega, a}\right\rangle_{\omega}-\left[\frac{1}{k !} \int\left(K_{y}^{\alpha}\right)^{(k)}\left(m_{J}\right) d \mu(y)\right]\left\langle x^{k}, h_{J}^{\omega, a}\right\rangle_{\omega}\right| \\
\leq & \frac{1}{k !}\left|\left\langle\left[\int_{\theta \in J} \sup _{\theta}\left|\left(K_{y}^{\alpha}\right)^{(k)}(\theta)-\left(K_{y}^{\alpha}\right)^{(k)}\left(m_{J}^{k}\right)\right| d \mu(y)\right]\left|x-m_{J}^{k}\right|^{k},\left|h_{J}^{\omega, a}\right|\right\rangle_{\omega}\right| \\
\lesssim & C_{C Z} \frac{\mathrm{P}_{k+\delta}^{\alpha}(J,|\mu|)}{|J|^{k}}\left\|\left(x-m_{J}^{k}\right)^{k}\right\|_{L^{2}\left(\mathbf{1}_{J} \omega\right)}
\end{aligned}
$$

where in the last line we have used

$$
\begin{aligned}
& \int \sup _{\theta \in J}\left|\left(K_{y}^{\alpha}\right)^{(k)}(\theta)-\left(K_{y}^{\alpha}\right)^{(k)}\left(m_{J}^{k}\right)\right| d \mu(y) \\
\lesssim & C_{C Z} \int\left(\frac{|J|}{\left|y-c_{J}\right|}\right)^{\delta} \frac{d|\mu|(y)}{\left|y-c_{J}\right|^{k+1-\alpha}}=C_{C Z} \frac{\mathrm{P}_{k+\delta}^{\alpha}(J, \mu)}{|J|^{k}} .
\end{aligned}
$$


Thus we have

$$
\begin{aligned}
\left\|\triangle_{J}^{\omega} T^{\alpha} \mu\right\|_{L^{2}(\omega)}^{2} & =\sum_{a \in \Gamma(J)}\left|\left\langle T^{\alpha} \mu, h_{J}^{\omega, a}\right\rangle_{\omega}\right|^{2} \\
= & \left|\frac{1}{k !} \int\left(K_{y}^{\alpha}\right)^{(k)}\left(m_{J}\right) d \mu(y)\right|^{2} \sum_{a \in \Gamma(J)}\left|\left\langle x^{k}, h_{J}^{\omega, a}\right\rangle_{\omega}\right|^{2} \\
& +O\left(\frac{\mathrm{P}_{k+\delta}^{\alpha}(J, \mu)}{|J|^{k}}\right)^{2}\left\|\left(x-m_{J}^{k}\right)^{k}\right\|_{L^{2}\left(\mathbf{1}_{J} \omega\right)}^{2}
\end{aligned}
$$

and hence

$$
\begin{aligned}
& c_{1}\left(\frac{1}{k !} \int\left(K_{y}^{\alpha}\right)^{(k)}\left(m_{J}\right) d \mu(y)\right)^{2}\left\|\triangle_{J}^{\omega} x^{k}\right\|_{L^{2}(\omega)}^{2}-C_{2}\left(\frac{\mathrm{P}_{k+\delta}^{\alpha}(J,|\mu|)}{|J|^{k}}\right)^{2}\left\|\left(x-m_{J}^{k}\right)^{k}\right\|_{L^{2}\left(\mathbf{1}_{J} \omega\right)}^{2} \\
\leq & \left\|\triangle_{J}^{\omega} T^{\alpha} \mu\right\|_{L^{2}(\omega)}^{2} \\
\leq & C_{1}\left(\frac{1}{k !} \int\left(K_{y}^{\alpha}\right)^{(k)}\left(m_{J}\right) d \mu(y)\right)^{2}\left\|\triangle_{J}^{\omega} x^{k}\right\|_{L^{2}(\omega)}^{2}+C_{2}\left(\frac{\mathrm{P}_{k+\delta}^{\alpha}(J,|\mu|)}{|J|^{k}}\right)^{2}\left\|\left(x-m_{J}^{k}\right)^{k}\right\|_{L^{2}\left(\mathbf{1}_{J} \omega\right)}^{2}
\end{aligned}
$$

where

$$
\left|\frac{1}{k !} \int\left(K_{y}^{\alpha}\right)^{(k)}\left(m_{J}\right) d \mu(y)\right| \approx \frac{\mathrm{P}_{k}^{\alpha}(J, \mu)}{|J|^{k}} .
$$

4.2. Comparison of $k$-energy and the usual 1-energy. We can write

$$
\left(\frac{\mathrm{P}_{k}^{\alpha}\left(J, \mathbf{1}_{I} \sigma\right)}{|J|^{k}}\right)^{2}\left\|\left(x-m_{J}^{k}\right)^{k}\right\|_{L^{2}\left(\mathbf{1}_{J} \omega\right)}^{2}=\mathrm{P}_{k}^{\alpha}\left(J, \mathbf{1}_{I} \sigma\right)^{2}\left\|\left(\frac{x-m_{J}^{k}}{|J|}\right)^{k}\right\|_{L^{2}\left(\mathbf{1}_{J} \omega\right)}^{2}
$$

and clearly we have the inequalities

$$
\begin{aligned}
\mathrm{P}_{k}^{\alpha}\left(J, \mathbf{1}_{I} \sigma\right) & =\int_{\mathbb{R}} \frac{|J|^{k}}{\left(|J|+\left|y-c_{J}\right|\right)^{k+1-\alpha}} d \sigma(y) \\
& =\int_{\mathbb{R}}\left(\frac{|J|}{\left(|J|+\left|y-c_{J}\right|\right)}\right)^{k-\ell} \frac{|J|^{\ell}}{\left(|J|+\left|y-c_{J}\right|\right)^{\ell+1-\alpha}} d \sigma(y) \\
& \leq \int_{\mathbb{R}} \frac{|J|^{\ell}}{\left(|J|+\left|y-c_{J}\right|\right)^{\ell+1-\alpha}} d \sigma(y)=\mathrm{P}_{\ell}^{\alpha}\left(J, \mathbf{1}_{I} \sigma\right),
\end{aligned}
$$

and

$$
\begin{aligned}
\left\|\left(\frac{x-m_{J}^{k}}{|J|}\right)^{k}\right\|_{L^{2}\left(\mathbf{1}_{J} \omega\right)}^{2} & \leq\left\|\left(\frac{x-m_{J}^{\ell}}{|J|}\right)^{k}\right\|_{L^{2}\left(\mathbf{1}_{J} \omega\right)}^{2}=\int_{J}\left(\frac{x-m_{J}^{\ell}}{|J|}\right)^{2 k} d \omega(x) \\
& \leq \int_{J}\left(\frac{x-m_{J}^{\ell}}{|J|}\right)^{2 \ell} d \omega(x)=\left\|\left(\frac{x-m_{J}^{\ell}}{|J|}\right)^{\ell}\right\|_{L^{2}\left(\mathbf{1}_{J} \omega\right)}^{2}
\end{aligned}
$$

for $1 \leq \ell \leq k$ since $\left|\frac{x-m_{J}^{\ell}}{|J|}\right| \leq 1$, and as a consequence we obtain

$$
\mathcal{E}_{2, k}^{\alpha} \leq \mathcal{E}_{2, \ell}^{\alpha}, \quad \text { for } 1 \leq \ell \leq k .
$$


4.3. An example with $\mathcal{A}_{2}^{0}<\infty, \mathcal{E}_{2, k}^{0}<\infty$ and $\mathcal{E}_{2,1}^{0}=\infty$. Define intervals

$$
I_{j} \equiv\left[\frac{1}{4^{j}}-\frac{1}{\sqrt{j} 4^{j}}, \frac{1}{4^{j}}\right], \quad j=2,3,4, \ldots
$$

and for $\varepsilon>0$ define measures

$$
\begin{aligned}
& d \sigma(y)=\delta_{0}+\sum_{j=2}^{\infty} \frac{4^{j}}{j^{\frac{1}{2}+\varepsilon}} \mathbf{1}_{I_{j}}(y) d y \\
& d \omega(x)=\sum_{j=2}^{\infty} \frac{j^{\frac{1}{2}}}{4^{j}} \mathbf{1}_{I_{j}}(x) d x
\end{aligned}
$$

so that

$$
\begin{aligned}
\left|I_{j}\right| & =\frac{1}{\sqrt{j} 4^{j}} \\
\left|I_{j}\right|_{\sigma} & =\frac{4^{j}}{j^{\frac{1}{2}+\varepsilon}} \frac{1}{\sqrt{j} 4^{j}}=\frac{1}{j^{1+\varepsilon}}, \\
\left|I_{j}\right|_{\omega} & =\frac{j^{\frac{1}{2}}}{4^{j}} \frac{1}{\sqrt{j} 4^{j}}=\frac{1}{4^{2 j}} .
\end{aligned}
$$

Then we compute that

$$
\begin{aligned}
\frac{\left|I_{j}\right|_{\sigma}\left|I_{j}\right|_{\omega}}{\left|I_{j}\right|^{2}} & =\frac{\frac{1}{j^{1+\varepsilon}} \frac{1}{4^{2 j}}}{\left(\frac{1}{\sqrt{j^{j}}}\right)^{2}}=\frac{1}{j^{\varepsilon}}, \\
\frac{\left|\left[0, \frac{1}{4^{j}}\right]\right|_{\sigma}\left|\left[0, \frac{1}{4^{j}}\right]\right|_{\omega}}{\left|\left[0, \frac{1}{4^{j}}\right]\right|^{2}} & =\frac{\left(1+\sum_{i=j}^{\infty}\left|I_{i}\right|_{\sigma}\right)\left(\sum_{i=j}^{\infty} \frac{1}{4^{2 i}}\right)}{\left(\frac{1}{4^{j}}\right)^{2}} \approx 1,
\end{aligned}
$$

and in fact it can be verified that $\mathcal{A}_{2}(\sigma, \omega) \approx 1$. We also have

$$
\left\|\frac{x-m_{I_{j}}^{1}}{\left|I_{j}\right|}\right\|_{L^{2}\left(\mathbf{1}_{I_{j}} \omega\right)}^{2}=\int_{I_{j}}\left(\frac{x-m_{I_{j}}^{1}}{\left|I_{j}\right|}\right)^{2} d \omega(x) \approx\left|I_{j}\right|_{\omega}=\frac{1}{4^{2 j}}
$$

and writing $\mathrm{P}=\mathrm{P}_{1}^{0}$ with $I=[0,1]$, we have

$$
\begin{aligned}
\mathrm{P}\left(I_{j}, \mathbf{1}_{I} \sigma\right) & =\int_{I} \frac{\left|I_{j}\right|}{\left[\left|I_{j}\right|+\left|y-c_{I_{j}}\right|\right]^{2}} d \sigma(y) \approx \frac{\left|I_{j}\right|}{\left[\left|I_{j}\right|+\left|0-c_{I_{j}}\right|\right]^{2}}+\int_{I_{j}} \frac{\left|I_{j}\right|}{\left[\left|I_{j}\right|+\left|y-c_{I_{j}}\right|\right]^{2}} d \sigma(y) \\
& \approx \frac{\frac{1}{\sqrt{j} 4^{j}}}{\left[\frac{1}{4^{j}}\right]^{2}}+\frac{\left|I_{j}\right|_{\sigma}}{\left|I_{j}\right|}=\frac{4^{j}}{\sqrt{j}}+\frac{\frac{1}{j^{1+\varepsilon}}}{\frac{1}{\sqrt{j^{j}}}}=\frac{4^{j}}{j^{\frac{1}{2}}}+\frac{4^{j}}{j^{\frac{1}{2}+\varepsilon}} \approx \frac{4^{j}}{j^{\frac{1}{2}}},
\end{aligned}
$$

where we note that the delta mass $\delta_{0}$ in $\sigma$ contributes the dominant term. From this we compute

$$
\mathcal{E}_{2,1}^{\alpha} \geq \frac{1}{|I|_{\sigma}} \sum_{j=2}^{\infty} \mathrm{P}\left(I_{j}, \mathbf{1}_{I} \sigma\right)^{2}\left\|\frac{x-m_{I_{j}}^{1}}{\left|I_{j}\right|}\right\|_{L^{2}\left(\mathbf{1}_{I_{j}} \omega\right)}^{2} \approx \sum_{j=2}^{\infty}\left(\frac{4^{j}}{j^{\frac{1}{2}}}\right)^{2} \frac{1}{4^{2 j}}=\sum_{j=2}^{\infty} \frac{1}{j}=\infty .
$$

On the other hand, writing $\mathrm{P}_{k}=\mathrm{P}_{k}^{0}$ we have

$$
\begin{aligned}
\mathrm{P}_{k}\left(I_{j}, \mathbf{1}_{I} \sigma\right) & =\int_{I}\left(\frac{\left|I_{j}\right|}{\left(\left|I_{j}\right|+\left|y-c_{I_{j}}\right|\right)}\right)^{k-1} \frac{\left|I_{j}\right|}{\left[\left|I_{j}\right|+\left|y-c_{I_{j}}\right|\right]^{2}} d \sigma(y) \\
& \approx\left(\frac{\left|I_{j}\right|}{\left(\left|I_{j}\right|+\left|0-c_{I_{j}}\right|\right)}\right)^{k-1} \frac{\left|I_{j}\right|}{\left[\left|I_{j}\right|+\left|0-c_{I_{j}}\right|\right]^{2}}+\int_{I_{j}} \frac{\left|I_{j}\right|}{\left[\left|I_{j}\right|+d\left(y, I_{j}\right)\right]^{2}} d \sigma(y) \\
& \approx\left(\frac{\frac{1}{\sqrt{j} 4^{j}}}{\frac{1}{4^{j}}}\right)^{k-1} \frac{\frac{1}{\sqrt{j} 4^{j}}}{\left[\frac{1}{4^{j}}\right]^{2}}+\frac{\left|I_{j}\right|_{\sigma}}{\left|I_{j}\right|} \approx \frac{4^{j}}{j^{\frac{k}{2}}}+\frac{\frac{1}{j^{1+\varepsilon}}}{\frac{1}{\sqrt{j} 4^{j}}} \approx \frac{4^{j}}{j^{\frac{1}{2}+\varepsilon}},
\end{aligned}
$$


where when $k \geq 2$, the dominant term arises from that part of $\sigma$ supported on $I_{j}$, and hence is significantly smaller than $\mathrm{P}\left(I_{j}, \mathbf{1}_{I} \sigma\right)$. Then for $k \geq 2$ we compute

$$
\frac{1}{|I|_{\sigma}} \sum_{j=2}^{\infty} \mathrm{P}_{k}\left(I_{j}, \mathbf{1}_{I} \sigma\right)^{2}\left\|\left(\frac{x-m_{I_{j}}^{k}}{\left|I_{j}\right|}\right)^{k}\right\|_{L^{2}\left(\mathbf{1}_{I_{j}} \omega\right)}^{2} \lesssim \sum_{j=2}^{\infty}\left(\frac{4^{j}}{j^{\frac{1}{2}+\varepsilon}}\right)^{2} \frac{1}{4^{2 j}}=\sum_{j=2}^{\infty} \frac{1}{j^{1+2 \varepsilon}}<\infty,
$$

and it can be shown that in fact $\mathcal{E}_{2, k}^{\alpha}<\infty$ by considering arbitrary decompositions $I=\dot{\cup}_{r=1}^{\infty} I_{r}$.

4.4. A Calderón-Zygmund operator satisfying testing conditions . We do not have an example of a weight pair $(\sigma, \omega)$, and a familiar Calderón-Zygmund operator $T$, to which Conjecture 16] applies, and to which the known T1-type theorems fail to apply. Our purpose here is to instead construct a rather artificial example to demonstrate that Conjecture 16] is at least 'logically' different than the known T1-type theorems in [LaWi] and SaShUr7.

For this we first construct a dyadic operator $T_{(\sigma, \omega)}^{\mathrm{dy}}$ that always satisfies testing conditions for a given weight pair $(\sigma, \omega)$. For an arbitrary weight pair $(\sigma, \omega)$ define

$$
T_{(\sigma, \omega)}^{\mathrm{dy}} f \equiv \sum_{I \in \mathcal{D}} \sum_{1 \leq i, j \leq 2}\left\langle f, a_{I}^{\sigma, i}\right\rangle_{L^{2}(\sigma)} a_{I}^{\omega, j}
$$

where $\left\{a_{I}^{\sigma, 1}, a_{I}^{\sigma, 2}\right\}_{I \in \mathcal{D}}$ and $\left\{a_{I}^{\omega, 1}, a_{I}^{\omega, 2}\right\}_{I \in \mathcal{D}}$ are Alpert bases for $L^{2}(\sigma)$ and $L^{2}(\omega)$ respectively. Then for any interval $Q \in \mathcal{D}$ and polynomial $p$ of degree at most 1 we have

$$
\begin{aligned}
\int_{Q}\left|T_{(\sigma, \omega)}^{\mathrm{dy}} \mathbf{1}_{Q} p\right|^{2} d \omega & =\int_{Q}\left|\sum_{I \in \mathcal{D}} \sum_{1 \leq i, j \leq 2}\left\langle\mathbf{1}_{Q} p, a_{I}^{\sigma, i}\right\rangle_{L^{2}(\sigma)} a_{I}^{\omega, j}\right|^{2} d \omega \\
& =\sum_{I \in \mathcal{D}} \sum_{1 \leq j \leq 2} \int_{Q}\left|\sum_{1 \leq i \leq 2}\left\langle\mathbf{1}_{Q} p, a_{I}^{\sigma, i}\right\rangle_{L^{2}(\sigma)}\right|^{2}\left|a_{I}^{\omega, j}\right|^{2} d \omega \\
& =\sum_{I \in \mathcal{D}: I \nsupseteq Q} \sum_{1 \leq j \leq 2} \int_{Q}\left|\widehat{\mathbf{1}_{Q} p}(I)\right|^{2}\left|a_{I}^{\omega, j}\right|^{2} d \omega \\
& =\sum_{I \in \mathcal{D}: I \nsupseteq Q}\left|\widehat{\mathbf{1}_{Q} p}(I)\right|^{2} \leq \int_{Q}|p|^{2} d \sigma .
\end{aligned}
$$

Similarly we have the dual testing condition.

To construct a Calderón-Zygmund operator $T$ that satisfies the testing conditions for $(\sigma, \omega)$ and whose kernel is $(k+\delta)$-smooth as in (4.1) for any $k+\delta>1$, we choose a subgrid $\mathcal{D}^{\prime}$ of $\mathcal{D}$ satisfying:

$$
\text { if } I, J \in \mathcal{D}^{\prime} \text { satisfy }|I| \leq|J| \text { and } \frac{11}{9} I \cap \frac{11}{9} J \neq \emptyset \text {, then } \frac{10}{9} I \subset J,
$$

and define functions $a_{I}^{\sigma}$ and $a_{I}^{\omega}$ to satisfy the following conditions with $\mu$ equal $\sigma$ and $\omega$ :

$$
\begin{aligned}
\operatorname{Supp} a_{I}^{\mu} & \subset \frac{11}{9} I, \\
0 & \leq a_{I}^{\mu}(x) \leq c|I|^{-\frac{1}{2}} \text { for } x \in \mathbb{R}, \\
a_{I}^{\mu}(x) & =c \text { for } x \in \frac{10}{9} I, \\
\int\left|a_{I}^{\mu}\right|^{2} d \mu & =1, \\
\int_{\mathbb{R}} x a_{I}^{\mu}(x) d \mu(x) & =\int_{\mathbb{R}} a_{I}^{\mu}(x) d \mu(x)=0, \\
\left|\frac{d^{\ell}}{d x^{\ell}} a_{I}^{\mu}(x)\right| & \leq C_{\ell}|I|^{-\frac{1}{2}-\ell}, \quad \ell \geq 0 .
\end{aligned}
$$


Note that these functions are not Alpert functions, but that they do form an orthonormal collection in $L^{2}(\mu)$. Then we set

$$
T f=\sum_{I \in \mathcal{D}^{\prime}}\left\langle f, a_{I}^{\sigma}\right\rangle_{L^{2}(\sigma)} a_{I}^{\omega}
$$

and note that $T$ is a $(k+\delta)$-smooth Calderón-Zygmund operator (see below) that satisfies the testing conditions for the weight pair $(\sigma, \omega)$ by the same argument above that established the testing conditions for the dyadic operator $T_{(\sigma, \omega)}^{\mathrm{dy}}$. If we take the weight pair $(\sigma, \omega)$ constructed above, then Conjecture 16 gives the boundedness of $T$ from $L^{2}(\sigma)$ to $L^{2}(\omega)$.

Lemma 20. The operator $T$ defined above is a $(k+\delta)$-smooth 0-fractional standard Calderón-Zymund operator for all $k+\delta>1$.

Proof. Note that the kernel is $K(x, y)=\sum_{I \in \mathcal{D}^{\prime}} a_{I}^{\omega}(x) a_{I}^{\sigma}(y)$. Assume that $|x-y| \simeq \frac{11}{9} 2^{-\ell}$ (i.e. $\frac{11}{9} 2^{-\ell} \lesssim$ $\left.|x-y| \lesssim \frac{11}{9} 2^{-\ell+1}\right)$. The sum in the expression of $K(x, y)$ only contains those terms which correspond to the intervals in $\mathcal{D}^{\prime}$ for which $x, y \in \frac{11}{9} I$. The smallest such interval has length $2^{-\ell}$. Also, for $m=0,1, \ldots, \ell-3$ there are at most two intervals of length $2^{-\ell+m}$ for which $a_{I}^{\omega}(x) a_{I}^{\sigma}(y) \neq 0$. Thus there are at most $2(\ell-2)$ terms in the sum for $K(x, y)$.

Also, using the properties of $a_{I}^{\omega}$ and $a_{I}^{\sigma}$ there holds:

$$
\begin{aligned}
&\left|a_{I}^{\omega}(x) a_{I}^{\sigma}(y)\right| \lesssim|I|^{-\frac{1}{2}}|I|^{-\frac{1}{2}}=|I|^{-1}, \\
&\left|\frac{\partial}{\partial x} a_{I}^{\omega}(x) a_{I}^{\sigma}(y)\right| \lesssim|I|^{-\frac{1}{2}-1}|I|^{-\frac{1}{2}}=|I|^{-2}, \\
&\left|\frac{\partial}{\partial y} a_{I}^{\omega}(x) a_{I}^{\sigma}(y)\right| \lesssim|I|^{-\frac{1}{2}}|I|^{-\frac{1}{2}-1}=|I|^{-2}, \\
&\left|\nabla^{k} a_{I}^{\omega}(x) a_{I}^{\sigma}(y)\right| \leq C_{k}|I|^{-1-k},
\end{aligned}
$$

for all $k \geq 1$, and where $\nabla=\left(\frac{\partial}{\partial x}, \frac{\partial}{\partial y}\right)$.

We now use these estimates to show that the kernel $K(x, y)$ is a $(k+\delta)$-smooth 0 -fractional CZ kernel for all $k+\delta>1$. In fact it is well known (using the mean value theorem) that it suffices to show

$$
|\nabla K(x, y)| \leq C_{k} \frac{1}{|x-y|^{k+1}}, \quad \text { for all } k \geq 0 .
$$

Recalling that $|x-y| \simeq \frac{11}{9} 2^{-\ell}$ we have for $k \geq 0$,

$$
\begin{aligned}
\left|\nabla^{k} K(x, y)\right| & \leq C_{k} \sum_{I \in \mathcal{D}^{\prime}} \sum_{i+j=k}\left|\left(\nabla_{x}^{i} a_{I}^{\omega}(x)\right)\left(\nabla_{y}^{j} a_{I}^{\sigma}(y)\right)\right| \leq C_{k} \sum_{I \in \mathcal{D}^{\prime}}|I|^{-k-1} \\
& \leq C_{k} \sum_{m=0}^{\ell-3} 2^{(k+1)(\ell-m)} \approx C_{k} 2^{\ell(k+1)} \approx C_{k} \frac{1}{|x-y|^{k+1}} .
\end{aligned}
$$

Remark 21. However, we can write this measure $\sigma$ as $\delta_{0}+\widetilde{\sigma}$ and note that the weight pair $(\widetilde{\sigma}, \omega)$ satisfies the usual energy condition (the 1-energy condition), and so $T$ is bounded from $L^{2}(\widetilde{\sigma})$ to $L^{2}(\omega)$ by results in either LaWi] or SaShUr7, and hence

$$
\int|T f \sigma|^{2} d \omega \lesssim \int|T f \widetilde{\sigma}|^{2} d \omega+\int\left|T f \delta_{0}\right|^{2} d \omega \lesssim \int|f|^{2} d \widetilde{\sigma}+|f(0)|^{2} \int\left|\sum_{I \in \mathcal{D}^{\prime}} a_{I}^{\sigma}(0) a_{I}^{\omega}\right|^{2} d \omega .
$$

Since $|f(0)|^{2}=\int|f|^{2} d \delta_{0} \leq \int|f|^{2} d \sigma$, it now remains only to prove that

$$
\int\left|\sum_{I \in \mathcal{D}^{\prime}} a_{I}^{\sigma}(0) a_{I}^{\omega}\right|^{2} d \omega=\sum_{I \in \mathcal{D}^{\prime}}\left|a_{I}^{\sigma}(0)\right|^{2} \lesssim 1
$$

which in turn holds simply because there is at most one $I \in \mathcal{D}^{\prime}$ containing the origin, and for such an interval we have that $\left|a_{I}^{\sigma}(0)\right| \lesssim 1$ due to the presence of the unit point mass at the origin, a much better 
bound than the general bound $c|I|^{-\frac{1}{2}}$. Indeed, the only intervals $I \in \mathcal{D}$ that contain the origin are the intervals $K_{j}=\left[0,4^{-j}\right)$ for some integer $j$, and by 4.5) there is at most one of these in $\mathcal{D}^{\prime}$ containing the origin. Thus we see that the Calderón-Zygmund operator $T$ constructed above is very artificial. Nonetheless,

it does show that Conjecture 16] contains boundedness results not included in [LaWi] and [SaShUr7], and potentially some not so trivial as that above as well.

\section{REFERENCES}

[Alp] Bradley K. Alpert, A class of bases in $L^{2}$ for the sparse representation of integral operators, SIAM J. Math. Anal 1 (1993), p. 246-262.

[CoJoSe] R. R. Coifman, P. W. Jones And S. Semmes, Two elementary proofs of the L2 boundedness of Cauchy integrals on Lipschitz curves, Journal of the A.M.S. 2 (1989), p. 553-564.

[DaJoSe] G. David, J.-L. Journé, And S. Semmes, Opérateurs de Calderón-Zygmund, fonctions para-accrétives et interpolation. Rev. Mat. Iberoamericana 1 (1985), 1-56.

[Hyt2] Hytönen, TuOmas, The two weight inequality for the Hilbert transform with general measures, arXiv:1312.0843v2.

[Lac] Michael T. LACEY, Two weight inequality for the Hilbert transform: A real variable characterization, II, Duke Math. J. Volume 163, Number 15 (2014), 2821-2840.

[LaSaShUr3] Michael T. Lacey, Eric T. Sawyer, Chun-Yen Shen, and Ignacio Uriarte-Tuero, Two weight inequality for the Hilbert transform: A real variable characterization I, Duke Math. J, Volume 163, Number 15 (2014), $2795-2820$.

[LaWi] Michael T. Lacey and Brett D. Wick, Two weight inequalities for Riesz transforms: uniformly full dimension weights, arXiv:1312.6163.

[NTV4] F. NAzArov, S. Treil AND A. Volberg, Two weight estimate for the Hilbert transform and corona decomposition for non-doubling measures, preprint (2004) arxiv:1003.1596.

[SaShUr7] Eric T. Sawyer, Chun-Yen Shen, and Ignacio Uriarte-Tuero, A two weight theorem for $\alpha$-fractional singular integrals with an energy side condition, Revista Mat. Iberoam. 32 (2016), no. 1, 79-174.

[SaShUr9] Sawyer, Eric T., Shen, Chun-Yen, Uriarte-Tuero, Ignacio, A two weight fractional singular integral theorem with side conditions, energy and $k$-energy dispersed, Harmonic Analysis, Partial Differential Equations, Complex Analysis, Banach Spaces, and Operator Theory (Volume 2) (Celebrating Cora Sadosky's life), Springer 2017 (see also arXiv: 1603.04332v2).

[SaShUr10] Sawyer, Eric T., Shen, Chun-Yen, Uriarte-Tuero, Ignacio, A good- $\lambda$ lemma, two weight T1 theorems without weak boundedness, and a two weight accretive global Tb theorem, Harmonic Analysis, Partial Differential Equations and Applications (In Honor of Richard L. Wheeden), Birkhäuser 2017 (see also arXiv:1609.08125v2).

[Vol] A. Volberg, Calderón-Zygmund capacities and operators on nonhomogeneous spaces, CBMS Regional Conference Series in Mathematics (2003), MR 2019058 (2005c:42015)\}. 